

\title{
Steady Flow of a Navier-Stokes Liquid Past an Elastic Body
}

\author{
Giovanni P. Galdi \\ Department of Mechanical Engineering and Materials Science \\ University of Pittsburgh, U.S.A \\ email:galdi@engr.pitt.edu \\ Mads Kyed \\ Institut für Mathematik \\ RWTH-Aachen, Germany \\ email:kyed@instmath.rwth-aachen.de
}

December 11, 2007

\begin{abstract}
We perform a mathematical analysis of the steady flow of a viscous liquid, $\mathcal{L}$, past a three-dimensional elastic body, $\mathcal{B}$. We assume that $\mathcal{L}$ fills the whole space exterior to $\mathcal{B}$, and that its motion is governed by the Navier-Stokes equations corresponding to non-zero velocity at infinity, $\mathbf{v}_{\infty}$. As for $\mathcal{B}$, we suppose that it is a St.Venant-Kirchoff material, held in equilibrium either by keeping an interior portion of it attached to a rigid body, or by means of appropriate control body force and surface traction. We treat the problem as a coupled steady state fluid-structure problem with the surface of $\mathcal{B}$ as a free boundary. Our main goal is to show existence and uniqueness for the coupled system liquid-body, for sufficiently small $\left|\mathbf{v}_{\infty}\right|$. This goal is reached by a fixed point approach based upon a suitable reformulation of the Navier-Stokes equation in the reference configuration, along with appropriate a priori estimates of solutions to the corresponding Oseen linearization and to the elasticity equations.
\end{abstract}

\section{Introduction}

The rigorous study of the problem of a coupled system constituted by a liquid interacting with an elastic structure is a relatively new branch of applied mathematics. In fact, the first significant contributions, due to ANTMAN AND LANZA De CRISTOForis, date back only to the early nineties; see $[15,16,1]$. In these papers the authors establish several properties related to the two-dimensional, irrotational steady flow of an inviscid liquid past nonlinear elastic bodies of different types. 
Also in view of the central role that the above problem plays in numerous and diverse engineering [2] and medical [11] applications, more recently mathematicians have begun a systematic study of the interaction of a Navier-Stokes (viscous) liquid with elastic bodies, in both steady [17, 12, 18] and unsteady $[13,4,6,7]$ cases. In all these works, the liquid occupies a bounded region of the three-dimensional space, that surrounds (or is surrounded by) the elastic structure.

In the present paper we would like to furnish a further contribution to the subject, by investigating the problem of steady state, three-dimensional flow of a Navier-Stokes liquid past an elastic body, kept in place by suitable mechanisms. We believe that, among other things, this study will be useful to set the appropriate function-analytic framework for further investigations, like, for example, bifurcation problems related to buckling of the elastic body due to the impingement of the liquid upon its surface. ${ }^{1}$

We shall now describe our problem in more precise terms.

Let $\mathcal{B}$ be an elastic body fully submerged in a Navier-Stokes liquid, $\mathcal{L}$, whose velocity field tends to a nonzero constant vector, $\mathbf{v}_{\infty}$, at large distance from $\mathcal{B}$. In the frame, $\mathcal{I}$, with respect to which we describe the motion of $\mathcal{L}$, we suppose that $\mathcal{B}$ is in equilibrium. Thus, in order to balance the forces exerted by $\mathcal{L}$ on $\mathcal{B}$, we assume either that $\mathcal{B}$ is attached to (and completely sourrounds) a non-deformable body, $\mathcal{R}$, fixed in $\mathcal{I}$, or that control forces are being applied to $\mathcal{B}$. Furthermore, we assume that $\mathcal{L}$ fills the whole space outside $\mathcal{B}$ and that the motion of $\mathcal{L}$ in $\mathcal{I}$ is steady.

The main objective of this paper is to investigate unique solvability for this problem, under the assumption that $\left|\mathbf{v}_{\infty}\right|$ is sufficiently small.

In the case where $\mathcal{B}$ is attached to $\mathcal{R}$, we assume they together occupy a bounded domain, $\Omega \subset \mathbb{R}^{3}$, when no applied body or surface forces act on them. We shall denote by $\omega \subset \Omega$ the part occupied by $\mathcal{R}$ and by $\Omega_{0}=\Omega \backslash \bar{\omega}$ that occupied by $\mathcal{B}$. We assume that $\mathcal{B}$ completely sourrounds $\mathcal{R}$, so that $\partial \omega \cap \partial \Omega=\emptyset$. We shall refer to $\Omega_{0}$ as the reference domain of the elastic body and suppose that $\Omega_{0}$ and $\omega$ are of class $C^{2}$. For simplicity, we contemplate the case where $\omega$ is a (bounded) domain ${ }^{2}$, and will refer to a coordinate system with the origin at some point $0 \in \omega$. We also set $\Gamma_{0}:=\partial \Omega$ and $\Gamma_{1}:=\partial \omega$; see Figure 1 .

If we denote by $\boldsymbol{\Phi}: \Omega_{0} \rightarrow \mathbb{R}^{3}$ the resulting deformation of $\mathcal{B}$ due to the forces exerted on it by the steady flow $(\mathbf{v}, p)$ past it, we find that the motion of the coupled system liquid-solid is governed by the following equations, in the case

\footnotetext{
${ }^{1}$ For steady bifurcation results of a steady-state flow of a Navier-Stokes liquid past a rigid body we refer to [10].

${ }^{2}$ The case where $\omega$ is the union of more than one domain does not introduce any conceptual difficulty.
} 


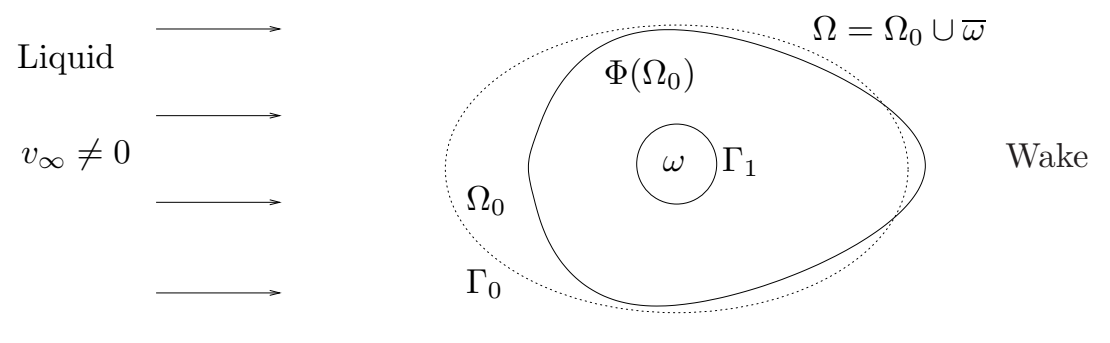

Figure 1: Flow past an elastic body attached to a non-deformable body.

where the elastic body is attached to $\omega \neq \emptyset$ :

$$
\begin{aligned}
& \left\{\begin{array}{rlrl}
-\nu \Delta \mathbf{v}+(\nabla \mathbf{v}) \mathbf{v}+\nabla p & =0 & & \text { in } \mathbb{R}^{3} \backslash\left(\boldsymbol{\Phi}\left(\Omega_{0}\right) \cup \omega\right) \\
\operatorname{div}(\mathbf{v}) & =0 & & \text { in } \mathbb{R}^{3} \backslash\left(\boldsymbol{\Phi}\left(\Omega_{0}\right) \cup \omega\right), \\
\mathbf{v} & =0 & & \text { on } \partial\left(\boldsymbol{\Phi}\left(\Omega_{0}\right)\right) \backslash \partial \omega, \\
\lim _{|\mathbf{x}| \rightarrow \infty} \mathbf{v} & =\mathbf{v}_{\infty}, &
\end{array}\right. \\
& \left\{\begin{aligned}
-\operatorname{div}\left(\mathbf{T}_{E}^{\mathbf{\Phi}}\right) & =0 & & \text { in } \boldsymbol{\Phi}\left(\Omega_{0}\right) \\
\mathbf{n}^{\mathbf{\Phi}} \mathbf{T}_{E}^{\mathbf{\Phi}} & =\mathbf{n}^{\mathbf{\Phi}} \mathbf{T}_{F}(\mathbf{v}, p) & & \text { on } \partial\left(\boldsymbol{\Phi}\left(\Omega_{0}\right)\right) \backslash \partial \omega \\
\boldsymbol{\Phi} & =\mathrm{Id} & & \text { on } \partial \omega
\end{aligned}\right.
\end{aligned}
$$

Here $\nu$ denotes the (constant) coefficient of kinematical viscosity of the liquid, $\mathbf{n}^{\mathbf{\Phi}}$ the outward normal on $\partial\left(\boldsymbol{\Phi}\left(\Omega_{0}\right)\right), \mathbf{T}_{E}^{\mathbf{\Phi}}$ the Cauchy stress tensor of the elastic material (the constitutive equations for which are determined by the assumption that the material is of St.Venant-Kirchoff type, see (24)), and $\mathbf{T}_{F}(\mathbf{v}, p)$ the Cauchy stress tensor of the liquid,

$$
\begin{aligned}
& \mathbf{T}_{F}(\mathbf{v}, p):=2 \nu \mathbf{D}(\mathbf{v})-p \mathbf{I}, \\
& \mathbf{D}(\mathbf{v}):=\frac{1}{2}\left(\nabla \mathbf{v}+\nabla \mathbf{v}^{\top}\right),
\end{aligned}
$$

where $\mathbf{I}$ is the identity tensor. Recall that for solenoidal velocities

$$
\operatorname{div} \mathbf{T}_{F}(\mathbf{v}, p)=\nu \Delta \mathbf{v}-\nabla p
$$

We observe that the boundary condition $\mathbf{n}^{\mathbf{\Phi}} \mathbf{T}_{E}^{\Phi}=\mathbf{n}^{\mathbf{\Phi}} \mathbf{T}_{F}(\mathbf{v}, p)$, on the liquidstructure interface, states that the force exerted by the elastic body on the liquid is the opposite of the force exerted by the liquid on the body. Furthermore, the boundary condition $\boldsymbol{\Phi}=\mathrm{Id}$ on $\partial \omega$ in (2) describes the attachment of the elastic body on $\omega$. We shall consider the problem where $\mathbf{v}_{\infty}$ is given and the deformation $\boldsymbol{\Phi}$ together with the liquid flow $(\mathbf{v}, p)$ are the unknowns. Note that the above model is only meaningful when there is no contact between $\partial \omega$ and $\partial \boldsymbol{\Phi}\left(\Omega_{0}\right)$, which will, however, be the case if the magnitude of $\mathbf{v}_{\infty}$ is sufficiently small. 


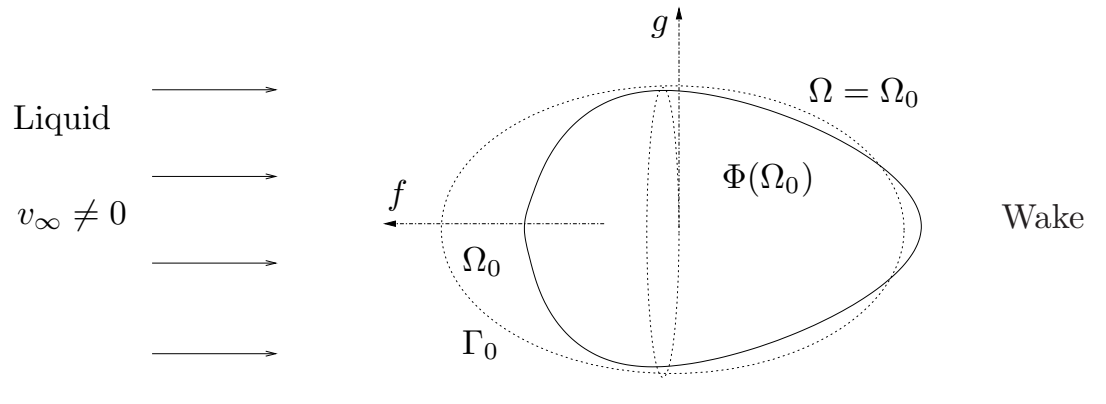

Figure 2: Flow past an elastic body with control forces $f$ and $g$.

In the case where the elastic body is kept in place by control forces, $\mathbf{f}$, and surface tractions, $\mathbf{g}$, we shall use the same notation as above and simply assume that $\omega=\emptyset$. In principle, the control parameters $\mathbf{f}$ and $\mathbf{g}$ can be chosen in an variety of ways. We shall make the following "simplest" choice

$$
\mathbf{f}=\mathbf{c}, \mathbf{g}=\mathbf{k} \wedge \mathbf{n}
$$

where $\mathbf{c}, \mathbf{k} \in \mathbb{R}^{3}$ and $\mathbf{n}$ is the unit outer normal to $\Omega_{0}$; see Figure 2 . Of course, the vectors $\mathbf{c}$ and $\mathbf{k}$ are further unknowns of the problem. Note that our choice of control parameters in (3) are expressed as forces in the reference domain $\Omega_{0}$ and $\partial \Omega_{0}$. Thus, the equations governing the equilibrium of $\mathcal{B}$ in this case are

$$
\left\{\begin{aligned}
-\operatorname{div}\left(\mathbf{T}_{E}^{\boldsymbol{\Phi}}\right) & =\left(\operatorname{det} \nabla \boldsymbol{\Phi}^{-1}\right) \mathbf{c} & & \text { in } \boldsymbol{\Phi}\left(\Omega_{0}\right), \\
\mathbf{n}^{\boldsymbol{\Phi}} \mathbf{T}_{E}^{\boldsymbol{\Phi}} & =\mathbf{n}^{\boldsymbol{\Phi}} \mathbf{T}_{F}(\mathbf{v}, p)+\mathbf{k} \wedge\left(\operatorname{cof} \nabla \boldsymbol{\Phi}^{-1} \mathbf{n}^{\mathbf{\Phi}}\right) & & \text { on } \partial\left(\boldsymbol{\Phi}\left(\Omega_{0}\right)\right),
\end{aligned}\right.
$$

while those governing the motion of $\mathcal{L}$ remain the same, and we are led to study the coupled systems (1) and (4).

For simplicity, in the models above we have assumed that all prescribed body forces, $\boldsymbol{b}$, and surface tractions, $\boldsymbol{t}$, are equal to zero. In fact, our results, suitably restated, continue to hold with non-zero $\boldsymbol{b}$ and $\boldsymbol{t}$. Also, we have chosen to consider the no-slip boundary condition $\mathbf{v}=0$ on the liquid-structure surface, as this is the most interesting situation from a physical point of view. Without further difficulties, however, more general boundary conditions could be handled.

Our main result is a proof of existence and uniqueness of solutions for the coupled systems (1), (2) and for the coupled systems (1), (4). More precisely, we show that if $\left|\mathbf{v}_{\infty}\right|$ is below a certain quantity, depending on $\Omega_{0}$ and on the material constants characterizing $\mathcal{L}$ and $\mathcal{B}$, there is one and only one solution lying in a ball, centered at the origin of a suitable Banach space, whose radius continuously depends on $\left|\mathbf{v}_{\infty}\right|$.

The above result is obtained by a fixed point contraction argument based on a priori estimates for proper linearizations of the governing equations. Being $\mathbf{v}_{\infty} \neq 0$, the "natural" linearization of the Navier-Stokes equations is provided 
by the Oseen equations. Actually, the basic challenge of our approach resides in finding the appropriate function space, $\mathfrak{S}$, where the estimates to the fully non-homogeneous, exterior Oseen boundary-value problem need to be proved. It turns out that $\mathfrak{S}$ can be chosen as the intersection of suitable Lebesgue and homogeneous Sobolev spaces; see Lemma 4.1.

Without loss of generality, we shall take $\mathbf{v}_{\infty}$ directed along the unit vector $\mathbf{e}_{1}$ and write $\mathbf{v}_{\infty}=v_{\infty} \mathbf{e}_{1}$ with $v_{\infty}>0$.

Remark 1.1. The arbitrary constant up to which the pressure $p$ is defined in (1) is fixed by requiring that $p(\mathbf{x}) \rightarrow 0$ as $|\mathbf{x}| \rightarrow \infty$. In other words, we fix the constant equal to zero. We could fix the constant to be any non-zero number, $p_{0}$, if we adjust our assumptions on the reference configuration accordingly. More precisely, since $p_{0}$ determines the force exerted by the liquid on the elastic body when the liquid is at rest, the reference configuration must be assumed to be the domain occupied by the elastic body when the force determined by $p_{0}$ is exerted on it.

Remark 1.2. If $\omega=\emptyset$, that is, if the body does not have a clamped portion of its boundary, and we were not to introduce any control forces, the liquid-structure problem would not have a solution. Actually, if $\omega=\emptyset$ it follows that

$$
\begin{aligned}
0=\int_{\boldsymbol{\Phi}\left(\Omega_{0}\right)} \operatorname{div}\left(\mathbf{T}_{E}^{\mathbf{\Phi}} \mathbf{e}_{1}\right) \mathrm{d} y & =\int_{\partial \boldsymbol{\Phi}\left(\Omega_{0}\right)} \mathbf{n}^{\mathbf{\Phi}} \mathbf{T}_{E}^{\mathbf{\Phi}} \mathbf{e}_{1} \mathrm{~d} S \\
& =\int_{\partial \boldsymbol{\Phi}\left(\Omega_{0}\right)} \mathbf{n}^{\boldsymbol{\Phi}} \mathbf{T}_{F}(\mathbf{v}, p) \mathbf{e}_{1} \mathrm{~d} S .
\end{aligned}
$$

This relation shows that the component of the force exerted by the liquid on the body in the direction of $\mathbf{v}_{\infty}$ (the drag) is zero, a condition that can not be verified (see [9, Theorem IX.5.1]).

The plan of the paper is the following. In Section 2 we introduce the basic notation and prove some preparatory results. Successively, in Section 3, we reformulate the liquid-body problem in the reference configuration. In Section 4 we prove existence, uniqueness, and fundamental estimates for the exterior Oseen boundary-value problem in proper function spaces. With the aid of these results, we prove similar ones for the fully non-linear exterior Navier-Stokes boundary-value problem. In Section 5, we recall a well-known well-posedness theorem for the elasticity equations in Sobolev spaces and prove further estimates for corresponding solutions. Finally, in Section 6, we combine the results of the previous two sections and use the fixed point contraction lemma to show existence and (local) uniqueness of solutions to the stated liquid-solid problems. 


\section{Notation and Preliminary Considerations.}

We begin to recall some classical notation and differential identities. If $\mathbf{A}, \mathbf{B}$ denote second-order tensors in $\mathbb{R}^{3}$, we set ${ }^{3}$

$$
\mathbf{A} \mathbf{B}=A_{i l} B_{l j} \mathbf{e}_{i} \otimes \mathbf{e}_{j},
$$

where $A_{i k}$ and $B_{l j}, i, j, l, k=1,2,3$, are the components of $\mathbf{A}$ and $\mathbf{B}$ in the canonical base, $\left\{\mathbf{e}_{1}, \mathbf{e}_{2}, \mathbf{e}_{3}\right\}$, of $\mathbb{R}^{3}$, and $\otimes$ denotes dyadic product. Moreover, if $\mathbf{a}$ is a vector with components $a_{i}, i=1,2,3$, in that base, we set $\mathbf{A} \mathbf{a}=A_{i k} a_{k} \mathbf{e}_{i}$ and $\mathbf{a} \mathbf{A}=A_{i k} a_{i} \mathbf{e}_{k}$. We also define

$$
\operatorname{div} \mathbf{A}=\frac{\partial A_{i j}}{\partial x_{i}} \mathbf{e}_{j}, \quad \nabla \mathbf{a}=\frac{\partial a_{i}}{\partial x_{k}} \mathbf{e}_{i} \otimes \mathbf{e}_{k} .
$$

We shall use boldface letters to denote vectors and tensors as well as vectorand tensor-valued functions in $\mathbb{R}^{3}$.

Let $\chi: \mathbf{x} \in \mathbb{R}^{3} \mapsto \mathbf{y}=\chi(\mathbf{x}) \in \mathbb{R}^{3}$ be a diffeomorphism of class $C^{1}$ from $\mathbb{R}^{3}$ onto itself. Thus, setting

$$
\mathbf{F}:=\nabla \chi, \quad J:=\operatorname{det} \mathbf{F}
$$

we have (see for example [3])

$$
\begin{aligned}
& \operatorname{div}\left(J \mathbf{F}^{-1} \mathbf{A}\right)=J \operatorname{div}_{y} \mathbf{A}, \\
& \operatorname{div}\left(J \mathbf{F}^{-1} \mathbf{a}\right)=J \operatorname{div}_{y} \mathbf{a}, \\
& (\nabla \mathbf{a}) \mathbf{F}^{-1}=\nabla_{y} \mathbf{a} .
\end{aligned}
$$

If, in (5), we choose $\mathbf{A}=\mathbf{I}$, with $\mathbf{I}$ denoting the identity tensor, we obtain the Piola identity

$$
\operatorname{div}\left(J \mathbf{F}^{-1}\right)=0 .
$$

We shall denote by $W^{m, q}(\Omega)$ the classical non-homogeneous Sobolev spaces and by $\|\cdot\|_{m, q, \Omega}$ the associated norm. By $D^{m, q}(\Omega)$ we denote the homogeneous Sobolev spaces defined by

$$
D^{m, q}(\Omega):=\left\{\left.u \in L_{l o c}^{1}(\Omega)|| u\right|_{m, q}:=\left(\sum_{|\alpha|=m} \int_{\Omega}\left|\mathrm{D}^{\alpha} u\right|^{q} \mathrm{~d} x\right)^{\frac{1}{q}}<\infty\right\} .
$$

We use $D_{0}^{1, q}(\Omega)$ to denote the completion of $C_{c}^{\infty}(\Omega)$ with respect to the norm $|\cdot|_{1, q}$. Moreover, we use $D^{-1, q}(\Omega)$ to denote the dual of the space $D_{0}^{1, q^{\prime}}(\Omega)$ with $1=\frac{1}{q}+\frac{1}{q^{\prime}}$. Finally, we shall denote by $C_{B}(\Omega)$ the subspace of $C(\Omega)$ of bounded functions.

Next consider $\mathbf{u} \in C^{1}\left(\bar{\Omega} ; \mathbb{R}^{3}\right)$ and define

$$
\Phi_{\mathbf{u}}: \mathbf{x} \in \Omega \mapsto \mathbf{x}+\mathbf{u}(\mathbf{x}) \in \mathbb{R}^{3} .
$$

\footnotetext{
${ }^{3}$ Throughout this paper, we shall use the summation convention over repeated indexes.
} 
We shall usually denote by $\mathbf{u}$ a displacement vector-field of $\Omega$, in which case we refer to $\boldsymbol{\Phi}_{\mathbf{u}}$ as the corresponding deformation of $\Omega$. We now seek to construct, based on $\mathbf{u}$, a mapping deforming the exterior domain $\mathcal{E}:=\mathbb{R}^{3} \backslash \Omega$ accordingly. To this end, set

$$
S_{q, M}(\Omega):=\left\{\mathbf{u} \in W^{2, q}(\Omega) \mid\|\mathbf{u}\|_{2, q, \Omega} \leq M\right\} .
$$

The following lemma holds.

Lemma 2.1. Let $q>3$ and let $\Omega \subset \mathbb{R}^{3}$ be a bounded, Lipschitz domain. Moreover, let $B_{R} \supset \bar{\Omega}$ and set $\delta:=\operatorname{dist}\left(\partial B_{R}, \partial \Omega\right)$. Then, there is a $K_{0}=$ $K_{0}(q, \Omega, \delta)>0{ }^{4}$ such that for any $\mathbf{u} \in S_{q, M}(\Omega)$ with $0 \leq M<K_{0}$ we can find a function $\tilde{\mathbf{u}} \in W^{2, q}\left(\mathbb{R}^{3}\right) \cap C^{1}\left(\mathbb{R}^{3}\right)$ satisfying

$$
\begin{aligned}
& \tilde{\mathbf{u}}=\mathbf{u} \quad \text { in } \bar{\Omega}, \\
& \tilde{\mathbf{u}}(\mathbf{x})=0 \quad \text { for all } \mathbf{x} \in \mathbb{R}^{3} \backslash B_{R}, \\
& \|\tilde{\mathbf{u}}\|_{2, q, \mathbb{R}^{3}} \leq C_{0}\|\mathbf{u}\|_{2, q, \Omega} \quad \text { with } C_{0}=C_{0}(q, \Omega, \delta), \\
& \chi_{\mathbf{u}}(\mathbf{x}):=\mathbf{x}+\tilde{\mathbf{u}} \text { is a } C^{1} \text {-diffeomorphism from } \mathcal{E} \text { onto } \mathbb{R}^{3} \backslash \mathbf{\Phi}_{\mathbf{u}}(\Omega) .
\end{aligned}
$$

Proof. For any given $\mathbf{u} \in S_{q, M}(\Omega)$, we construct the extension $\tilde{\mathbf{u}}$ as follows. Let $\mathbf{U}$ be an extension of $\mathbf{u}$ to $\mathbb{R}^{3}$ with $\mathbf{U} \in W^{2, q}\left(\mathbb{R}^{3}\right)$. By classical results, we know that $\mathbf{U}$ exists and that

$$
\|\mathbf{U}\|_{2, q, \mathbb{R}^{3}} \leq c_{0}\|\mathbf{u}\|_{2, q, \Omega}
$$

with $c_{0}=c_{0}(q, \Omega)>0$. Let us next choose $r<R$ such that $\bar{\Omega} \subset B_{r} \subset B_{R}$, with $R-r=\delta / 2$, and let $\psi=\psi(\mathbf{x})$ be a smooth cut-off function that is equal to 1 for $\mathbf{x} \in B_{r}$ and is zero for $\mathbf{x} \in B_{R}^{c}$. This function can be chosen in such a way that $|D \psi(\mathbf{x})| \leq c_{1} \delta^{-1},\left|D^{2} \psi(\mathbf{x})\right| \leq c_{2} \delta^{-2}$, where $c_{i}, i=1,2$, are independent of $\delta, R$ and $r$. The desired extension is then given by $\tilde{\mathbf{u}}:=\psi \mathbf{U}$. In fact, (8) and (9) are obviously satisfied, whereas (10) is a consequence of (12) and of the properties of the function $\psi$. Consider now the map

$$
\chi_{\mathbf{u}}: \mathbf{x} \in \mathbb{R}^{3} \mapsto \mathbf{y}=\mathbf{x}+\tilde{\mathbf{u}} \in \mathbb{R}^{3} .
$$

By the Sobolev embedding theorem we have $W^{2, q}\left(\mathbb{R}^{3}\right) \hookrightarrow C^{1}\left(\mathbb{R}^{3}\right)$, and that

$$
\|\tilde{\mathbf{u}}\|_{C^{1}\left(\mathbb{R}^{3}\right)} \leq c_{3}\|\tilde{\mathbf{u}}\|_{2, q, \mathbb{R}^{3}}
$$

with $c_{3}=c_{3}(q)>0$. From this, it follows, in particular, that $\chi_{\mathbf{u}} \in C^{1}\left(\mathbb{R}^{3}\right)$. Furthermore, from (10) and from the fact that $\mathbf{u} \in S_{q, M}\left(\Omega_{0}\right)$, we obtain

$$
\|\tilde{\mathbf{u}}\|_{C^{1}\left(\mathbb{R}^{3}\right)} \leq c_{4} M
$$

and so, since

$$
\nabla \chi_{\mathbf{u}}=\mathbf{I}+\nabla \tilde{\mathbf{u}}
$$

\footnotetext{
${ }^{4} K_{0} \rightarrow 0$ as $\delta \rightarrow 0$.
} 
we have

$$
\begin{aligned}
\operatorname{det}\left(\nabla \chi_{\mathbf{u}}(\mathbf{x})\right) & =\prod_{j=1}^{3}\left(1+\partial_{j} \tilde{u}_{j}\right)+\sum_{\sigma \in S_{3} \backslash\{\operatorname{Id}\}} \prod_{j=1}^{3}(\mathbf{I}+\nabla \tilde{u})_{j \sigma(j)} \\
& =1+\sum_{j \in \mathcal{A}} \mathcal{M}_{j}(\nabla \tilde{\mathbf{u}})
\end{aligned}
$$

with $\mathcal{M}_{j}(\nabla \tilde{\mathbf{u}})$ a mononomial with respect to the entries of $\nabla \tilde{\mathbf{u}}$ and $|\mathcal{A}|=12$. Thus, if the constant $K_{0}$ is chosen as small as to satisfy the condition

$$
K_{0}<\frac{1}{12 c_{4}}
$$

then

$$
\operatorname{det}\left(\nabla \chi_{\mathbf{u}}(\mathbf{x})\right)>1-12 c_{4} K_{0}>0 .
$$

From [5, Theorem 5.5-1(b)] and (13)-(14), we then find that $\chi_{\mathbf{u}}$ is injective. In fact, it is easy to show that $\chi_{\mathbf{u}}$ is surjective which, in turn, since $\chi_{\mathbf{u}}=\boldsymbol{\Phi}_{\mathbf{u}}$ on $\bar{\Omega}$, implies that $\chi_{\mathbf{u}}$ is a diffeomorphism of class $C^{1}$ of $\mathcal{E}$ onto $\mathbb{R}^{3} \backslash \boldsymbol{\Phi}_{\mathbf{u}}(\Omega)$. To show surjectivity, for any fixed $\mathbf{y} \in \mathbb{R}^{3}$, consider the map

$$
\mathbf{P}: \mathbf{x} \in \mathbb{R}^{3} \mapsto \mathbf{y}-\tilde{\mathbf{u}}(\mathbf{x}) \in \mathbb{R}^{3} .
$$

We have, by (13), that $\mathbf{P} \in C^{1}\left(\mathbb{R}^{3}\right)$ and moreover, by (15), that

$$
\sup _{\mathbf{x} \in \mathbb{R}^{3}}|\nabla \mathbf{P}(\mathbf{x})|<1 .
$$

Therefore, by well-known results (see for example [14, Theorem 1.XVII]) it follows that $\mathbf{P}$ has a fixed point. This proves that $\chi_{\mathbf{u}}$ is surjective, and the proof of the lemma is thereby completed.

Throughout this paper we shall use small letters $\left(c_{0}, c_{1}, \ldots\right)$ to denote constants in scope of a single proof, and capital letters $\left(C_{0}, C_{1}, \ldots\right)$ to denote constants in scope of the whole paper.

For any exterior domain $\mathcal{O}$ we set $\mathcal{O}_{R}:=\mathcal{O} \cap B_{R}(0)$ and $\mathcal{O}^{R}:=\mathcal{O} \backslash B_{R}(0)$. Similarly, for any Banach space $\mathcal{X}$ we put $\mathcal{X}_{R}:=\{x \in \mathcal{X} \mid\|x\| \leq R\}$.

We shall from now on fix $\Omega, \Omega_{0}$, and $\omega$ to be the $C^{2}$-domains from the introduction. Moreover, we set $\mathcal{E}:=\mathbb{R}^{3} \backslash \Omega$ and fix $R>0$ such that $B_{R}(0) \supset \bar{\Omega}$ and put $\delta:=\operatorname{dist}\left(\partial B_{R}(0), \partial \Omega\right)$.

We shall make use of the Landau symbols (Big-O and Little-o notation) in the sense that $f \in O(t)$ iff $|f| \leq C|t|$ as $t \rightarrow 0$ and $f \in o(t)$ iff $\frac{|f|}{|t|} \rightarrow 0$ as $t \rightarrow 0$.

\section{Reformulation of the Problem in the Refer- ence Configuration.}

In order to solve (1) coupled with either (2) or (4), we first transform the systems into equivalent systems over the reference domain. For this purpose we use the 
mapping constructed in Lemma 2.1. Writing the deformation as the identity plus a displacement field, $\boldsymbol{\Phi}=\mathrm{Id}+\mathbf{u}$, the corresponding mapping $\chi_{\mathbf{u}}$, from Lemma 2.1, maps the reference exterior domain $\mathcal{E}:=\mathbb{R}^{3} \backslash\left(\Omega_{0} \cup \omega\right)$ onto the deformed exterior domain $\mathbb{R}^{3} \backslash\left(\boldsymbol{\Phi}\left(\Omega_{0}\right) \cup \omega\right)$. Set $\mathbf{y}=\chi_{\mathbf{u}}(\mathbf{x})$,

$$
\mathbf{w}=\mathbf{w}(\mathbf{x}):=\mathbf{v}\left(\chi_{\mathbf{u}}(\mathbf{x})\right), \quad q=q(\mathbf{x}):=p\left(\chi_{\mathbf{u}}(\mathbf{x})\right),
$$

and

$$
\mathbf{F}_{\mathbf{u}}:=\nabla \chi_{\mathbf{u}}, \quad J_{\mathbf{u}}:=\operatorname{det} \mathbf{F}_{\mathbf{u}} .
$$

With the help of (5)-(7) we then find

$$
\begin{aligned}
& \left(\nabla_{y} \mathbf{v}\right) \mathbf{v}=(\nabla \mathbf{w}) \mathbf{F}_{\mathbf{u}}^{-1} \mathbf{w}, \\
& \mathbf{D}(\mathbf{v})=\frac{1}{2}\left[(\nabla \mathbf{w}) \mathbf{F}_{\mathbf{u}}^{-1}+\mathbf{F}_{\mathbf{u}}^{-\top}(\nabla \mathbf{w})^{\top}\right]:=\mathbf{D}_{\mathbf{u}}(\mathbf{w}), \\
& \operatorname{div}_{y} \mathbf{T}(\mathbf{v}, q)=J_{\mathbf{u}}^{-1} \operatorname{div}\left[2 \nu J_{\mathbf{u}} \mathbf{F}_{\mathbf{u}}^{-1} \mathbf{D}_{\mathbf{u}}(\mathbf{w})\right]-J_{\mathbf{u}}^{-1} \operatorname{div}\left(J_{\mathbf{u}} \mathbf{F}_{\mathbf{u}}^{-1} q I\right), \\
& \operatorname{div}\left(J_{\mathbf{u}} \mathbf{F}_{\mathbf{u}}^{-1} \nabla \mathbf{w} \mathbf{F}_{\mathbf{u}}^{-1}\right)=J_{\mathbf{u}} \nabla_{y} \operatorname{div}_{y} \mathbf{v} .
\end{aligned}
$$

Using (5), (6), (17), (19), and (20) we now obtain that the Navier-Stokes equations (1) can be written over the reference domain as

$$
\left\{\begin{array}{rlrl}
-\nu \operatorname{div}\left(\mathbf{A}_{\mathbf{u}} \mathbf{F}_{\mathbf{u}}^{-\top} \nabla \mathbf{w}^{\top}\right)+\operatorname{div}\left(\mathbf{A}_{\mathbf{u}} q \mathbf{I}\right)+(\nabla \mathbf{w}) \mathbf{A}_{\mathbf{u}} \mathbf{w}=0 & & \text { in } \mathcal{E}, \\
\operatorname{div}\left(\mathbf{A}_{\mathbf{u}} \mathbf{w}\right) & =0 & & \text { in } \mathcal{E}, \\
\mathbf{w} & =0 & & \text { on } \Gamma_{0} \\
\lim _{|x| \rightarrow \infty} \mathbf{w} & =\mathbf{v}_{\infty}, &
\end{array}\right.
$$

whereby

$$
\mathbf{A}_{\mathbf{u}}:=J_{\mathbf{u}} \mathbf{F}_{\mathbf{u}}^{-1}=\left(\operatorname{cof} \mathbf{F}_{\mathbf{u}}\right)^{\top} .
$$

When $q>3, W^{1, q}(\mathcal{E})$ is a Banach algebra. In this case the equations in (21) become well-defined in a Sobolev space setting for $\mathbf{u} \in W^{2, q}(\Omega)$ since the entries of $\mathbf{F}_{\mathbf{u}}$, and thereby also the those of $\mathbf{A}_{\mathbf{u}}$, then belong to $W_{l o c}^{1, q}(\mathcal{E})$ by Lemma 2.1 . Also note that the asymptotic limits at infinity of $\mathbf{w}$ and $\mathbf{v}$ are identical since $\chi_{\mathbf{u}}(\mathbf{x})=1$ for large $|\mathbf{x}|$.

Introducing the first Piola-Kirchhoff stress tensor $\boldsymbol{\sigma}(\mathbf{u})$, and using the Piola Identity, we can write the elasticity equation (2) over the reference domain as

$$
\left\{\begin{aligned}
-\operatorname{div}(\boldsymbol{\sigma}(\mathbf{u})) & =0 & & \text { in } \Omega_{0}, \\
\mathbf{n} \boldsymbol{\sigma}(\mathbf{u}) & =\mathbf{n} \mathbf{T}_{F}^{\mathbf{u}}(\mathbf{w}, q) & & \text { on } \Gamma_{0}, \\
\mathbf{u} & =0 & & \text { on } \Gamma_{1},
\end{aligned}\right.
$$

where $\mathbf{n}$ is the outward normal on $\partial \mathcal{E}$ and

$$
\mathbf{T}_{F}^{\mathbf{u}}(\mathbf{w}, q)=\mathbf{A}_{\mathbf{u}}\left(2 \nu \mathbf{D}_{\mathbf{u}}(\mathbf{w})-q \mathbf{I}\right) .
$$

Similarly, we can write (4) over the reference domain as

$$
\left\{\begin{aligned}
-\operatorname{div}(\boldsymbol{\sigma}(\mathbf{u})) & =\mathbf{c} & & \text { in } \Omega_{0}, \\
\mathbf{n} \boldsymbol{\sigma}(\mathbf{u}) & =\mathbf{n} \mathbf{T}_{F}^{\mathbf{u}}(\mathbf{w}, q)+\mathbf{k} \wedge \mathbf{n} & & \text { on } \Gamma_{0} .
\end{aligned}\right.
$$


We assume the elastic body is a St.Venant-Kirchoff material, for which the first Piola-Kirchhoff stress tensor takes the form

$$
\begin{aligned}
& \boldsymbol{\sigma}(\mathbf{u})=(\lambda(\operatorname{Tr} \mathbf{E}(\mathbf{u})) \mathbf{I}+2 \mu \mathbf{E}(\mathbf{u}))(\mathbf{I}+\nabla \mathbf{u})^{\top}, \text { with } \\
& \mathbf{E}(\mathbf{u})=\frac{1}{2}\left(\nabla \mathbf{u}^{\top}+\nabla \mathbf{u}+\nabla \mathbf{u}^{\top} \nabla \mathbf{u}\right)
\end{aligned}
$$

and $\lambda$ and $\mu$ denoting the Lamé constants. We can write the first Piola-Kirchhoff stress tensor above as a sum of a linear, bi-linear, and tri-linear response function,

$$
\boldsymbol{\sigma}(\mathbf{u})=\overline{\boldsymbol{\sigma}}_{\boldsymbol{L}}(\nabla \mathbf{u})+\overline{\boldsymbol{\sigma}}_{\boldsymbol{B} \boldsymbol{L}}(\nabla \mathbf{u}, \nabla \mathbf{u})+\overline{\boldsymbol{\sigma}}_{\boldsymbol{T} \boldsymbol{L}}(\nabla \mathbf{u}, \nabla \mathbf{u}, \nabla \mathbf{u}),
$$

with $\overline{\boldsymbol{\sigma}}_{\boldsymbol{L}}: \mathbb{R}^{3 \times 3} \rightarrow \mathbb{R}^{3 \times 3}$ linear, $\overline{\boldsymbol{\sigma}}_{B \boldsymbol{L}}: \mathbb{R}^{3 \times 3} \times \mathbb{R}^{3 \times 3} \rightarrow \mathbb{R}^{3 \times 3}$ bi-linear, and $\bar{\sigma}_{T L}: \mathbb{R}^{3 \times 3} \times \mathbb{R}^{3 \times 3} \times \mathbb{R}^{3 \times 3} \rightarrow \mathbb{R}^{3 \times 3}$ tri-linear. Note that $\bar{\sigma}_{L}$ is the response function for the classical stress tensor of linear elasticity.

In the following, we show existence of a solution for the coupled systems (1), (2) and the systems (1), (4) by solving the equivalent systems (21), (22) and (21), (23), respectively.

\section{The Liquid Equations}

In this section we shall show that the liquid equations (21) over the reference domain have a unique solution (in suitable function class), provided $\mathbf{u} \in S_{q, M}\left(\Omega_{0}\right)$ and $\mathbf{v}_{\infty}$ and $M$ are sufficiently small. We shall also establish some estimates for the corresponding solutions.

First we rewrite (21) into

$$
\left\{\begin{aligned}
-\nu \Delta \mathbf{w}+\nabla q+(\nabla \mathbf{w}) \mathbf{w} & =\mathcal{F}_{\mathbf{u}}(\mathbf{w}, q) & & \text { in } \mathcal{E}, \\
\operatorname{div}(\mathbf{w}) & =\mathcal{G}_{\mathbf{u}}(\mathbf{w}) & & \text { in } \mathcal{E}, \\
\mathbf{w} & =0 & & \text { on } \Gamma_{0}, \\
\lim _{|x| \rightarrow \infty} \mathbf{w} & =\mathbf{v}_{\infty}, & &
\end{aligned}\right.
$$

with

$$
\begin{aligned}
\mathcal{F}_{\mathbf{u}}(\mathbf{w}, q):=- & \nu \operatorname{div}\left(\left(\mathbf{I}-\mathbf{A}_{\mathbf{u}} \mathbf{F}_{\mathbf{u}}^{-\top}\right) \nabla \mathbf{w}^{\top}\right)+ \\
& \quad \operatorname{div}\left(q \mathbf{I}-\mathbf{A}_{\mathbf{u}} q \mathbf{I}\right)+(\nabla \mathbf{w})\left(\mathbf{I}-\mathbf{A}_{\mathbf{u}}\right) \mathbf{w}
\end{aligned}
$$

and

$$
\mathcal{G}_{\mathbf{u}}(\mathbf{w}):=\operatorname{div}\left(\left(\mathbf{I}-\mathbf{A}_{\mathbf{u}}\right) \mathbf{w}\right)=\left(\mathbf{I}-\mathbf{A}_{\mathbf{u}}\right): \nabla \mathbf{w} .
$$

The last equality above is due to the Piola Identity.

The purpose of rewriting the equations (21) into (26) is to have the Navier Stokes equations on the left hand side and a perturbation term on the righthand side. When the displacement, $\mathbf{u}$, is sufficiently small, the perturbation term is small and we can hope to solve the equations using a fixpoint argument. 
For this, we shall need a priori estimates of the operator on the left-hand side. In order to obtain these, we shall further reformulate the equations such that we have the Oseen linearization of the Navier Stokes equations on the left-hand side. More specifically, we put (in accordance with the assumption that $\mathbf{v}_{\infty}$ is directed along $\mathbf{e}_{1}$ )

$$
\mathbf{z}:=\mathbf{w}-\mathbf{v}_{\infty}, \quad \pi:=q, \quad \mathbf{v}_{\infty}=v_{\infty} \mathbf{e}_{1}
$$

and obtain

$$
\left\{\begin{aligned}
-\nu \Delta \mathbf{z}+v_{\infty} \partial_{1} \mathbf{z}+\nabla \pi & =-(\nabla \mathbf{z}) \mathbf{z}+\mathcal{F}_{\mathbf{u}}\left(\mathbf{z}+\mathbf{v}_{\infty}, \pi\right) & & \text { in } \mathcal{E} \\
\operatorname{div}(\mathbf{z}) & =\mathcal{G}_{\mathbf{u}}(\mathbf{z}) & & \text { in } \mathcal{E} \\
\mathbf{z} & =-\mathbf{v}_{\infty} & & \text { on } \Gamma_{0} \\
\lim _{|x| \rightarrow \infty} \mathbf{z} & =0 . & &
\end{aligned}\right.
$$

We have the following lemma on existence and a priori estimates of the Oseen equations in exterior domains.

Lemma 4.1. Let $\mathcal{O}$ be an exterior domain of class $C^{2}$ and $\mathcal{R}>0$. Assume that

$$
\begin{aligned}
& \mathbf{f} \in L^{r}(\mathcal{O}) \cap D_{0}^{-1,12 / 7}(\mathcal{O}), \\
& g \in W^{1, r}(\mathcal{O}) \cap L^{12 / 7}(\mathcal{O}) \cap D_{0}^{-1,12 / 7}(\mathcal{O}), \text { and } \\
& \mathbf{v}_{*} \in W^{2-1 / r, r}(\partial \mathcal{O}) \cap W^{5 / 12,12 / 7}(\partial \mathcal{O})
\end{aligned}
$$

for some $r \in(1, \infty)$. Then, the problem

$$
\left\{\begin{aligned}
-\Delta \mathbf{v}+\mathcal{R} \partial_{1} \mathbf{v}+\nabla p & =\mathbf{f} & & \text { in } \mathcal{O} \\
\operatorname{div} \mathbf{v} & =g & & \text { in } \mathcal{O} \\
\mathbf{v} & =\mathbf{v}_{*} & & \text { on } \partial \mathcal{O} \\
\lim _{|x| \rightarrow \infty} \mathbf{v} & =0 & &
\end{aligned}\right.
$$

has one and only one solution $(\mathbf{v}, p)$ such that

$$
(\mathbf{v}, p) \in D^{2, r}(\mathcal{O}) \cap D^{1,12 / 7}(\mathcal{O}) \cap L^{4}(\mathcal{O}) \cap L^{3}(\mathcal{O}) \times D^{1, r}(\mathcal{O}) \cap L^{12 / 7}(\mathcal{O})
$$

Moreover, for any arbitrarily fixed $\mathcal{R}_{0}>0$, there exists $C_{\Theta}=C_{\Theta}\left(\mathcal{O}, \mathcal{R}_{0}, r\right)>0$ such that for any $0<\mathcal{R} \leq \mathcal{R}_{0}$ the corresponding solution of (31) satisfies

$$
\begin{gathered}
|\mathbf{v}|_{2, r}+|\mathbf{v}|_{1,12 / 7}+\|\mathbf{v}\|_{4}+\mathcal{R}^{1 / 4}\|\mathbf{v}\|_{3}+|p|_{1, r}+\|p\|_{12 / 7} \leq \\
C_{\Theta}\left(\|\mathbf{f}\|_{r}+|\mathbf{f}|_{-1,12 / 7}+\|g\|_{1, r}+\|g\|_{12 / 7}+|g|_{-1,12 / 7}\right. \\
\left.+\left\|\mathbf{v}_{*}\right\|_{2-1 / r, r, \partial \mathcal{O}}+\left\|\mathbf{v}_{*}\right\|_{5 / 12,12 / 7, \partial \mathcal{O}}\right) .
\end{gathered}
$$

Proof. Uniqueness in the class (32) follows from standard results (see [8, Theorem VII.6.2] and [8, Exercise VII.6.2]). Existence of a solution in a larger 
class than (32) also follows from standard results (see for example [8, Theorem VII.7.2]). Thus we only need to verify that (33) holds. Consider first a solution of the whole space problem

$$
\left\{\begin{aligned}
-\Delta \mathbf{w}+\mathcal{R} \partial_{1} \mathbf{w}+\nabla q=\mathbf{f} & \text { in } \mathbb{R}^{3}, \\
\operatorname{div} \mathbf{w}=g & \text { in } \mathbb{R}^{3}, \\
\lim _{|x| \rightarrow \infty} \mathbf{w}=0, &
\end{aligned}\right.
$$

with data satisfying (28) with $\mathbb{R}^{3}$ as underlying domain instead of $\mathcal{O}$. From $[8$, Theorem VII.4.1] we obtain the estimate

$$
|\mathbf{w}|_{2, r, \mathbb{R}^{3}}+|q|_{1, r, \mathbb{R}^{3}} \leq c_{0}\left(\mathcal{R}_{0}\right)\left(\|\mathbf{f}\|_{r, \mathbb{R}^{3}}+\|g\|_{1, r, \mathbb{R}^{3}}\right)
$$

and from [8, Theorem VII.4.2] the estimate

$$
\begin{array}{r}
\mathcal{R}^{1 / 4}\|\mathbf{w}\|_{3}+|\mathbf{w}|_{1,12 / 7}+\|q\|_{12 / 7} \leq \\
c_{1}\left(|\mathbf{f}|_{-1,12 / 7}+\mathcal{R}|g|_{-1,12 / 7}+\|g\|_{12 / 7}\right) .
\end{array}
$$

Using the same technique (using cut-off functions) as in the proof of $[8$, Theorem VII.7.1] and [8, Theorem VII.7.2], we obtain from (34) and (35) that the solution of the exterior domain problem (31) satisfies the estimates

$$
|\mathbf{v}|_{2, r, \mathcal{O}^{\rho / 2}}+|p|_{1, r, \mathcal{O}^{\rho / 2}} \leq \quad \begin{gathered}
c_{2}\left(\mathcal{R}_{0}\right)\left(\|\mathbf{f}\|_{r}+\|g\|_{1, r}+\|\mathbf{v}\|_{1, r, \mathcal{O}_{\rho}}+\|p\|_{r, \mathcal{O}_{\rho}}\right)
\end{gathered}
$$

and

$$
\begin{aligned}
& \mathcal{R}^{1 / 4} \| \mathbf{v}\left\|_{3, \mathcal{O}^{\rho / 2}}+|\mathbf{v}|_{1,12 / 7, \mathcal{O} \rho / 2}+\right\| p \|_{12 / 7, \mathcal{O}^{\rho / 2}} \leq \\
& c_{3}\left(|\mathbf{f}|_{-1,12 / 7}+|\mathbf{v}|_{-1,12 / 7, \mathcal{O}_{\rho}}+|\nabla \mathbf{v}|_{-1,12 / 7, \mathcal{O}_{\rho}}+|p|_{-1,12 / 7, \mathcal{O}_{\rho}}\right. \\
&\left.+\mathcal{R}|\mathbf{v}|_{-1,12 / 7, \mathcal{O}_{\rho}}+\mathcal{R}|g|_{-1,12 / 7}+\|\mathbf{v}\|_{12 / 7, \mathcal{O}_{\rho}}+\|g\|_{12 / 7}\right)
\end{aligned}
$$

whereby the constant $\rho>0$ is chosen such that $\overline{\mathcal{O}}^{c} \subset B_{\rho / 2}$. Using now the embedding $L^{12 / 11}\left(\mathcal{O}_{\rho}\right) \hookrightarrow D_{0}^{-1,12 / 7}\left(\mathcal{O}_{\rho}\right)$ and the fact that $12 / 11 \leq 12 / 7$, it follows that $|\mathbf{v}|_{-1,12 / 7, \mathcal{O}_{\rho}} \leq c_{4}\|\mathbf{v}\|_{12 / 7, \mathcal{O}_{\rho}}$. Hence we can reduce (37) to

$$
\begin{gathered}
\mathcal{R}^{1 / 4}\|\mathbf{v}\|_{3, \mathcal{O} \rho / 2}+|\mathbf{v}|_{1,12 / 7, \mathcal{O} \rho / 2}+\|p\|_{12 / 7, \mathcal{O} \rho / 2} \leq \\
c_{5}\left(\mathcal{R}_{0}\right)\left(|\mathbf{f}|_{-1,12 / 7}+|g|_{-1,12 / 7}+\|g\|_{12 / 7}\right. \\
\left.+|p|_{-1,12 / 7, \mathcal{O}_{\rho}}+\|v\|_{12 / 7, \mathcal{O}_{\rho}}\right)
\end{gathered}
$$

Now we seek similar estimates over $\mathcal{O}_{\rho}$. By ellipticity of the Stokes operator (see [8, Theorem IV.6.1] and [8, Exercise IV.6.2]) we have

$$
\begin{aligned}
\|\mathbf{v}\|_{2, r, \mathcal{O}_{\rho}}+ & \|p\|_{1, r, \mathcal{O}_{\rho}} \leq c_{6}\left(\|\mathbf{f}\|_{r, \mathcal{O}_{\rho}}+\|g\|_{1, r, \mathcal{O}_{\rho}}+\left\|\mathbf{v}_{*}\right\|_{2-1 / r, r, \partial \mathcal{O}}\right. \\
& \left.+\|\mathbf{v}\|_{2-1 / r, r, \partial B_{\rho}}+\|\mathbf{v}\|_{r, \mathcal{O}_{\rho}}+\mathcal{R}\left\|\partial_{1} \mathbf{v}\right\|_{r, \mathcal{O}_{\rho}}+\|p\|_{r, \mathcal{O}_{\rho}}\right)
\end{aligned}
$$


and

$$
\begin{aligned}
&\|\mathbf{v}\|_{1,12 / 7, \mathcal{O}_{\rho}}+\|p\|_{12 / 7, \mathcal{O}_{\rho}} \leq \\
& c_{7}\left(\mathcal{R}_{0}\right)\left(|\mathbf{f}|_{-1,12 / 7, \mathcal{O}_{\rho}}+\|g\|_{12 / 7, \mathcal{O}_{\rho}}+\left\|\mathbf{v}_{*}\right\|_{5 / 12,12 / 7, \partial \mathcal{O}}\right. \\
&\left.+\|\mathbf{v}\|_{5 / 12,12 / 7, \partial B_{\rho}}+\|\mathbf{v}\|_{12 / 7, \mathcal{O}_{\rho}}+\|p\|_{-1,12 / 7, \mathcal{O}_{\rho}}\right) .
\end{aligned}
$$

By [8, Theorem II.3.4] we have

$$
\|\mathbf{v}\|_{2-1 / r, r, \partial B_{\rho}} \leq c_{8}\left(|\mathbf{v}|_{2, r, \mathcal{O} \rho / 2}+\|\mathbf{v}\|_{1, r, \mathcal{O}_{\rho}}\right)
$$

and

$$
\|\mathbf{v}\|_{5 / 12,12 / 7, \partial B_{\rho}} \leq c_{9}\left(|\mathbf{v}|_{1,12 / 7, \mathcal{O}^{\rho / 2}}+\|\mathbf{v}\|_{12 / 7, \mathcal{O}_{\rho}}\right) .
$$

By the embedding $W^{1,12 / 7}\left(\mathcal{O}_{\rho}\right) \hookrightarrow L^{4}\left(\mathcal{O}_{\rho}\right)$ we furthermore have

$$
\|\mathbf{v}\|_{3, \mathcal{O}_{\rho / 2}} \leq c_{10}\|\mathbf{v}\|_{1,12 / 7, \mathcal{O}_{\rho / 2}} .
$$

Finally, from the embedding $D^{1,12 / 7}(\mathcal{O}) \hookrightarrow L^{4}(\mathcal{O})$ we obtain

$$
\|\mathbf{v}\|_{4} \leq c_{11}|\mathbf{v}|_{1,12 / 7} \text {. }
$$

Combining now (36), (38), and (39)-(44) yields

$$
\begin{aligned}
&|\mathbf{v}|_{2, r}+|\mathbf{v}|_{1,12 / 7}+\|\mathbf{v}\|_{4}+\mathcal{R}^{1 / 4}\|\mathbf{v}\|_{3}+|p|_{1, r}+\|p\|_{12 / 7} \leq \\
& c_{12}\left(\mathcal{R}_{0}\right)\left(\|\mathbf{f}\|_{r}+|\mathbf{f}|_{-1,12 / 7}+\|g\|_{1, r}+\|g\|_{12 / 7}+|g|_{-1,12 / 7}\right. \\
&+\left\|\mathbf{v}_{*}\right\|_{2-1 / r, r, \partial \mathcal{O}}+\left\|\mathbf{v}_{*}\right\|_{5 / 12,12 / 7, \partial \mathcal{O}} \\
&\left.+\|\mathbf{v}\|_{1, r, \mathcal{O}_{\rho}}+\|p\|_{r, \mathcal{O}_{\rho}}+\|\mathbf{v}\|_{12 / 7, \mathcal{O}_{\rho}}+|p|_{-1,12 / 7, \mathcal{O}_{\rho}}\right) .
\end{aligned}
$$

We will now show that

$$
\begin{gathered}
\|\mathbf{v}\|_{1, r, \mathcal{O}_{\rho}}+\|p\|_{r, \mathcal{O}_{\rho}}+\|\mathbf{v}\|_{12 / 7, \mathcal{O}_{\rho}}+|p|_{-1,12 / 7, \mathcal{O}_{\rho}} \leq \\
C\left(\mathcal{R}_{0}\right)\left(\|\mathbf{f}\|_{r}+|\mathbf{f}|_{-1,12 / 7}+\|g\|_{1, r}+\|g\|_{12 / 7}+|g|_{-1,12 / 7}\right. \\
\left.+\left\|\mathbf{v}_{*}\right\|_{2-1 / r, r, \partial \mathcal{O}}+\left\|\mathbf{v}_{*}\right\|_{5 / 12,12 / 7, \partial \mathcal{O}}\right)
\end{gathered}
$$

which together with (45) implies (33). Assume there exists no constant $C\left(\mathcal{R}_{0}\right)$ such that (46) holds for all solutions of (31). This would imply the existence of sequences

$$
\left\{\mathbf{f}_{k}\right\},\left\{g_{k}\right\} \subset C_{c}^{\infty}(\overline{\mathcal{O}}), \quad\left\{\mathbf{v}_{* k}\right\} \subset W^{2-1 / r, r}(\partial \mathcal{O}) \cap W^{5 / 12,12 / 7}(\partial \mathcal{O}),
$$

and

$$
\left\{\mathcal{R}_{k}\right\} \subset\left(0, \mathcal{R}_{0}\right]
$$

such that, denoting by $\left\{\left(\mathbf{v}_{k}, p_{k}\right)\right\}$ the solutions of the Oseen problems

$$
\left\{\begin{aligned}
-\Delta \mathbf{v}_{k}+\mathcal{R} \partial_{1} \mathbf{v}_{k}+\nabla p_{k} & =\mathbf{f}_{k} & & \text { in } \mathcal{O}, \\
\operatorname{div} \mathbf{v}_{k} & =g_{k} & & \text { in } \mathcal{O}, \\
\mathbf{v}_{k} & =\mathbf{v}_{* k} & & \text { on } \partial \mathcal{O}, \\
\lim _{|x| \rightarrow \infty} \mathbf{v}_{k} & =0, & &
\end{aligned}\right.
$$


$(k=1,2, \ldots)$, the following conditions hold

$$
\begin{gathered}
\left\|\mathbf{f}_{k}\right\|_{r}+\left|\mathbf{f}_{k}\right|_{-1,12 / 7}+\left\|g_{k}\right\|_{1, r}+\left\|g_{k}\right\|_{12 / 7}+\left|g_{k}\right|_{-1,12 / 7} \\
+\left\|\mathbf{v}_{* k}\right\|_{2-1 / r, r, \partial \mathcal{O}}+\left\|\mathbf{v}_{* k}\right\|_{5 / 12,12 / 7, \partial \mathcal{O}} \leq 1 / k \\
\left\|\mathbf{v}_{k}\right\|_{1, r, \mathcal{O}_{\rho}}+\left\|\mathbf{v}_{k}\right\|_{12 / 7, \mathcal{O}_{\rho}}+\left\|p_{k}\right\|_{r, \mathcal{O}_{\rho}}+\left|p_{k}\right|_{-1,12 / 7, \mathcal{O}_{\rho}}=1,
\end{gathered}
$$

and there is $\mathcal{R} \in\left[0, \mathcal{R}_{0}\right]$ such that

$$
\lim _{k \rightarrow \infty} \mathcal{R}_{k}=\mathcal{R}
$$

Since $\left(\mathbf{v}_{k}, p_{k}\right)$ are solutions of (47), they satisfy (45). By (48) and (49) this implies that $\left(\mathbf{v}_{k}, p_{k}\right)$ are bounded in the norms on the left hand side of (45). Thus we can find subsequences, still denoted by $\left\{\left(\mathbf{v}_{k}, p_{k}\right)\right\}$, and functions $(\mathbf{v}, p)$ in the class (32) such that, as $k \rightarrow \infty$,

$$
\begin{aligned}
& D^{2} \mathbf{v}_{k} \rightarrow D^{2} \mathbf{v} \quad \text { and } \quad \nabla p_{k} \rightarrow \nabla p \quad \text { weakly in } L^{r}(\mathcal{O}) \\
& \mathbf{v}_{k} \rightarrow \mathbf{v} \quad \text { weakly in } L^{4}(\mathcal{O}), \quad \text { and } \\
& \nabla \mathbf{v}_{k} \rightarrow \nabla \mathbf{v} \quad \text { and } \quad p_{k} \rightarrow p \quad \text { weakly in } L^{12 / 7}(\mathcal{O})
\end{aligned}
$$

By standard compact embeddings of Sobolev spaces we further obtain

$$
\begin{array}{ll}
\mathbf{v}_{k} \rightarrow \mathbf{v} & \text { strongly in } W^{1, r}\left(\mathcal{O}_{\rho}\right) \text { and } L^{12 / 7}\left(\mathcal{O}_{\rho}\right), \\
p_{k} \rightarrow p & \text { strongly in } L^{r}\left(\mathcal{O}_{\rho}\right) \text { and } W^{-1,12 / 7}\left(\mathcal{O}_{\rho}\right) .
\end{array}
$$

From (47)-(51) we conclude that $(\mathbf{v}, p)$ and $\mathcal{R}$ satisfy the conditions

$$
\begin{array}{rlrl}
-\Delta \mathbf{v}+\mathcal{R} \partial_{1} \mathbf{v}+\nabla p & =0 & & \text { in } \mathcal{O}, \\
\operatorname{div} \mathbf{v} & =0 & & \text { in } \mathcal{O}, \\
\mathbf{v} & =0 & & \text { on } \partial \mathcal{O}, \\
\lim _{|x| \rightarrow \infty} \mathbf{v} & =0, & \\
\mathbf{v} \in D^{1,12 / 7}(\mathcal{O}), & p \in L^{12 / 7}(\mathcal{O})
\end{array}
$$

and

$$
\|\mathbf{v}\|_{1, r, \mathcal{O}_{\rho}}+\|\mathbf{v}\|_{12 / 7, \mathcal{O}_{\rho}}+\|p\|_{r, \mathcal{O}_{\rho}}+\|p\|_{-1,12 / 7, \mathcal{O}_{\rho}}=1 .
$$

However, by classical uniqueness theorems for the Oseen (if $\mathcal{R}>0$ ) and Stokes (if $\mathcal{R}=0$ ) exterior problems (see [8, Theorem VII.6.2, Exercise VII.6.2 and Theorem V.5.1]), (52) and (53) implies $\mathbf{v} \equiv 0$ and $p \equiv 0$, which contradicts (54). The proof of the lemma is thus completed.

In order to formulate the next results, we find it convenient to define a number of Banach spaces. For any given $v_{\infty}>0$, we set

$$
\mathcal{Z}^{q}(\mathcal{E}):=\left\{\mathbf{z} \in L_{l o c}^{1}(\mathcal{E}) \mid\|\mathbf{z}\|_{\mathcal{Z}^{q}}<\infty\right\}
$$


whereby

$$
\|\mathbf{z}\|_{\mathcal{Z}^{q}}:=|\mathbf{z}|_{2, q}+|\mathbf{z}|_{1,12 / 7}+\|\mathbf{z}\|_{4}+v_{\infty}^{1 / 4}\|\mathbf{z}\|_{3} .
$$

It is obvious that $(55)$ defines a norm in $\mathcal{Z}^{q}(\mathcal{E})$ and that $\mathcal{Z}^{q}(\mathcal{E})$, equipped with this norm, becomes a Banach space. Likewise, set

$$
\mathcal{P}^{q}(\mathcal{E}):=\left\{p \in L_{l o c}^{1}(\mathcal{E}) \mid\|p\|_{\mathcal{P}^{q}}<\infty\right\},
$$

whereby

$$
\|p\|_{\mathcal{P}^{q}}:=|p|_{1, q}+\|p\|_{12 / 7} .
$$

The space $\mathcal{P}^{q}(\mathcal{E})$ equipped with the norm (56) becomes a Banach space. We also set

$$
\mathcal{X}^{q}(\mathcal{E}):=\mathcal{Z}^{q}(\mathcal{E}) \times \mathcal{P}^{q}(\mathcal{E}),\|(\mathbf{z}, p)\|_{\mathcal{X}^{q}}:=\|\mathbf{z}\|_{\mathcal{Z}^{q}}+\|p\|_{\mathcal{P}^{q}} .
$$

Furthermore, we define

$$
\mathcal{Y}^{q}(\mathcal{E}):=\left\{\mathbf{f} \in L_{l o c}^{1}(\mathcal{E}) \mid\|\mathbf{f}\|_{\mathcal{Y}^{q}}<\infty\right\},
$$

whereby

$$
\|\mathbf{f}\|_{\mathcal{Y}^{q}}:=|\mathbf{f}|_{-1,12 / 7}+\|\mathbf{f}\|_{q}
$$

and

$$
\mathcal{Q}^{q}(\mathcal{E}):=\left\{g \in L_{l o c}^{1}(\mathcal{E}) \mid\|g\|_{\mathcal{Q}^{q}}<\infty\right\},
$$

whereby

$$
\|g\|_{\mathcal{Q}^{q}}:=|g|_{-1,12 / 7}+\|g\|_{12 / 7}+\|g\|_{1, q} .
$$

In order to solve (27), we need to estimate all the terms on the right hand side. We start with the convective term.

Lemma 4.2. Let $q>3$ and $\mathbf{z}_{1}, \mathbf{z}_{2} \in \mathcal{Z}^{q}(\mathcal{E})$. Then, there are constants $C_{1}=$ $C_{1}(\mathcal{E}, q)>0$ and $C_{2}=C_{2}(\mathcal{E})>0$ such that

$$
\begin{aligned}
& \left\|\left(\nabla \mathbf{z}_{1}\right) \mathbf{z}_{2}\right\|_{q} \leq C_{1}\left\|\mathbf{z}_{1}\right\|_{\mathcal{Z}^{q}}\left\|\mathbf{z}_{2}\right\|_{\mathcal{Z}^{q}} \\
& \left|\left(\nabla \mathbf{z}_{1}\right) \mathbf{z}_{2}\right|_{-1,12 / 7} \leq C_{2} v_{\infty}^{-1 / 4}\left\|\mathbf{z}_{1}\right\|_{\mathcal{Z}^{q}}\left\|\mathbf{z}_{2}\right\|_{\mathcal{Z}^{q}}
\end{aligned}
$$

Proof. We recall the property that, if $h \in L^{r}(\mathcal{E}) \cap D^{1, q}(\mathcal{E})$, for some $r \geq 1$, then $h \in L^{\infty}(\mathcal{E})$ and the following inequality holds

$$
\|h\|_{\infty} \leq c_{1}\left(\|h\|_{r}+|h|_{1, q}\right),
$$

where $c_{1}=c_{1}(\mathcal{E}, r, q)>0$ (see for example [8, Remark II.7.2]). Let $\mathbf{z} \in \mathcal{Z}^{q}(\mathcal{E})$. From (59), with $r=12 / 7$, it easily follows that

$$
\begin{aligned}
|\mathbf{z}|_{1, q} & \leq|\mathbf{z}|_{1,12 / 7}^{12 / 7 q}\|\nabla \mathbf{z}\|_{\infty}^{1-12 / 7 q} \\
& \leq c_{2}|\mathbf{z}|_{1,12 / 7}^{12 / 7 q}\left(|\mathbf{z}|_{1,12 / 7}+|\mathbf{z}|_{2, q}\right)^{1-12 / 7 q} \leq c_{3}|\mathbf{z}|_{\mathcal{Z}^{q}}
\end{aligned}
$$


with $c_{i}=c_{i}(\mathcal{E}, q)>0, i=2,3$. Using again (59), with $r=4$, and (60) we also deduce

$$
\|\mathbf{z}\|_{\infty} \leq c_{4}\left(\|\mathbf{z}\|_{4}+|\mathbf{z}|_{1, q}\right) \leq c_{5}\|\mathbf{z}\|_{\mathcal{Z}^{q}}
$$

where $c_{i}=c_{i}(\mathcal{E}, q)>0, i=4,5$. Thus, by (60) and (61), we find, for some $c_{6}=c_{6}(\mathcal{E}, q)>0$,

$$
\left\|\left(\nabla \mathbf{z}_{1}\right) \mathbf{z}_{2}\right\|_{q} \leq\left\|\mathbf{z}_{2}\right\|_{\infty}\left|\mathbf{z}_{1}\right|_{1, q} \leq c_{6}\left\|\mathbf{z}_{1}\right\|_{\mathcal{Z}^{q}}\left\|\mathbf{z}_{2}\right\|_{\mathcal{Z}^{q}}
$$

which proves (57).

Consider now $\psi \in D_{0}^{1,12 / 5}(\mathcal{E})$. By the Hölder inequality and by $(55)$, we obtain

$$
\begin{aligned}
\left|\left(\left(\nabla \mathbf{z}_{1}\right) \mathbf{z}_{2}, \psi\right)\right| & \leq\left\|\mathbf{z}_{2}\right\|_{3}\left|\mathbf{z}_{1}\right|_{1,12 / 7}\|\psi\|_{12} \\
& \leq v_{\infty}^{-1 / 4}\left\|\mathbf{z}_{1}\right\|_{\mathcal{Z}^{q}}\left\|\mathbf{z}_{2}\right\|\left\|_{\mathcal{Z}^{q}}\right\| \psi \|_{12} .
\end{aligned}
$$

Therefore, (58) follows from the latter and from the continuity of the embedding $D_{0}^{1,12 / 5}(\mathcal{E}) \hookrightarrow L^{12}(\mathcal{E})$.

Having estimated the convective term on the right-hand side of (27), we move on to the perturbation terms. To this end we need the following estimates.

Lemma 4.3. Let $q>3$ and $K_{0}$ be as in Lemma 2.1. For $\mathbf{u}_{1}, \mathbf{u}_{2} \in S_{q, M}\left(\Omega_{0}\right)$ with $M<M_{0}:=\min \left\{K_{0}, 1\right\}$ the following properties hold:

1. The entries of $\mathbf{F}_{\mathbf{u}_{i}}^{-1}$ and $\mathbf{A}_{\mathbf{u}_{i}}$ are in $W^{1, q}\left(B_{\rho}\right)$ for all $\rho>0(i=1,2)$.

2. There exists a constant $C_{3}=C_{3}\left(q, \Omega_{0}, K_{0}, R, \delta\right)>0$ such that

$$
\left\|\mathbf{I}-\mathbf{A}_{\mathbf{u}_{i}}\right\|_{1, q, \mathcal{E}}+\left\|\mathbf{I}-\mathbf{A}_{\mathbf{u}_{i}} \mathbf{F}_{\mathbf{u}_{i}}^{-\top}\right\|_{1, q, \mathcal{E}} \leq C_{3}\left\|\mathbf{u}_{i}\right\|_{2, q, \Omega_{0}}, i=1,2 .
$$

3. There exists a constant $C_{4}=C_{4}\left(q, \Omega_{0}, K_{0}, R, \delta\right)>0$ such that

$$
\begin{array}{r}
\left\|\mathbf{A}_{\mathbf{u}_{1}} \mathbf{F}_{\mathbf{u}_{1}}^{-\top}-\mathbf{A}_{\mathbf{u}_{2}} \mathbf{F}_{\mathbf{u}_{2}}^{-\top}\right\|_{1, q, \mathcal{E}}+\left\|\mathbf{A}_{\mathbf{u}_{1}}-\mathbf{A}_{\mathbf{u}_{2}}\right\|_{1, q, \mathcal{E}} \\
\leq C_{4}\left\|\mathbf{u}_{1}-\mathbf{u}_{2}\right\|_{2, q, \Omega_{0}} .
\end{array}
$$

Proof. Put $\mathbf{u}=\mathbf{u}_{i}, i=1,2$. By definition, $\mathbf{F}_{\mathbf{u}}=\mathbf{I}+\nabla \tilde{\mathbf{u}}$ and $\tilde{\mathbf{u}} \in W^{2, q}\left(\mathbb{R}^{3}\right)$. Since $q>3, W^{1, q}\left(B_{\rho}\right)$ is a Banach algebra, from which it follows that the entries of $\operatorname{cof}\left(\mathbf{F}_{\mathbf{u}}\right)$ and hence of $\mathbf{A}_{\mathbf{u}}$ are in $W^{1, q}\left(B_{\rho}\right)$. Moreover, by Lemma 2.1 the entries of $\mathbf{F}_{\mathbf{u}}$ are continuous, and from (16) we have the pointwise estimate

$$
\operatorname{det}\left(\mathbf{F}_{\mathbf{u}}\right)>c_{0}\left(K_{0}\right)>0 .
$$

Since

$$
\mathbf{F}_{\mathbf{u}}^{-1}=\frac{1}{\operatorname{det}\left(\mathbf{F}_{\mathbf{u}}\right)}\left(\operatorname{cof}\left(\mathbf{F}_{\mathbf{u}}\right)\right)^{\top}
$$


it follows that the entries of $\mathbf{F}_{\mathbf{u}}^{-1}$ are in $L^{q}\left(B_{\rho}\right)$. Denoting by inv : $\mathbb{G L}_{3,3}(\mathbb{R}) \rightarrow$ $\mathbb{M}_{3,3}(\mathbb{R})$ the inversion mapping $\operatorname{inv}(A)=A^{-1}$ and using that $\langle\partial \operatorname{inv}(A), H\rangle=$ $-A^{-1} H A^{-1}$, we find that

$$
\begin{aligned}
\partial_{i}\left[\mathbf{F}_{\mathbf{u}}^{-1}\right] & =\partial_{i}\left[\operatorname{inv}\left(\mathbf{F}_{\mathbf{u}}\right)\right] \\
& =-\mathbf{F}_{\mathbf{u}}^{-1}\left(\partial_{i} \mathbf{F}_{\mathbf{u}}\right) \mathbf{F}_{\mathbf{u}}^{-1}=\frac{1}{\operatorname{det}\left(\mathbf{F}_{\mathbf{u}}\right)^{2}}\left(\operatorname{cof} \mathbf{F}_{\mathbf{u}}\right)^{\top}\left(\partial_{i} \mathbf{F}_{\mathbf{u}}\right)\left(\operatorname{cof} \mathbf{F}_{\mathbf{u}}\right)^{\top} .
\end{aligned}
$$

Using (64) and the fact that the entries of $\operatorname{cof} \mathbf{F}_{\mathbf{u}}$ are in $C_{B}\left(B_{\rho}\right)$ (due to the embedding $\left.W^{1, q}\left(B_{\rho}\right) \hookrightarrow C_{B}\left(B_{\rho}\right)\right)$ we obtain that also the entries of $\partial_{i}\left[\mathbf{F}_{\mathbf{u}}^{-1}\right]$ are in $L^{q}\left(B_{\rho}\right)$. Thus we conclude that the entries of $\mathbf{F}_{\mathbf{u}}^{-1}$ are in $W^{1, q}\left(B_{\rho}\right)$.

We now estimate

$$
\begin{aligned}
\left\|\mathbf{I}-\mathbf{A}_{\mathbf{u}}\right\|_{1, q}^{2} & =\left\|\delta_{i j}-\operatorname{cof}(\mathbf{I}+\nabla \tilde{\mathbf{u}})_{j i}\right\|_{1, q}^{2} \\
& =\left\|1-\operatorname{cof}(\mathbf{I}+\nabla \tilde{\mathbf{u}})_{i i}\right\|_{1, q}^{2}+\sum_{i \neq j}\left\|\operatorname{cof}(\mathbf{I}+\nabla \tilde{\mathbf{u}})_{j i}\right\|_{1, q}^{2} \\
& =\left\|1-\operatorname{det}(\mathbf{I}+\nabla \tilde{\mathbf{u}})^{i i}\right\|_{1, q}^{2}+\sum_{i \neq j}\left\|\operatorname{det}(\mathbf{I}+\nabla \tilde{\mathbf{u}})^{j i}\right\|_{1, q}^{2} \\
& =\sum_{i=1}^{3}\left\|1-\left(1+\mathcal{M}_{i}(\nabla \tilde{\mathbf{u}})\right)\right\|_{1, q}^{2}+\sum_{i \neq j}\left\|\mathcal{M}_{i j}(\nabla \tilde{\mathbf{u}})\right\|_{1, q}^{2},
\end{aligned}
$$

with $\mathcal{M}(\nabla \tilde{\mathbf{u}})$ being a mononomial with respect to the entries of $\nabla \tilde{\mathbf{u}}$. Using that $\|\tilde{\mathbf{u}}\|_{2, q} \leq c_{1}\|\mathbf{u}\|_{2, q, \Omega_{0}}$ and the fact that, by assumption, $\|\mathbf{u}\|_{2, q, \Omega_{0}}<1$, we obtain

$$
\left\|\mathbf{I}-\mathbf{A}_{\mathbf{u}}\right\|_{1, q}^{2} \leq c_{2}\|\mathbf{u}\|_{2, q, \Omega_{0}}^{2}
$$

For the second term in (62) we have the estimate

$$
\begin{aligned}
\left\|\mathbf{I}-\mathbf{A}_{\mathbf{u}_{i}} \mathbf{F}_{\mathbf{u}_{i}}^{-\top}\right\|_{1, q} & =\left\|\left(\mathbf{F}_{\mathbf{u}}^{\top}-\mathbf{I}+\mathbf{I}-\mathbf{A}_{\mathbf{u}}\right) \mathbf{F}_{\mathbf{u}}^{-\top}\right\|_{1, q} \\
& \leq c_{3}\left(\left\|\mathbf{F}_{\mathbf{u}}-\mathbf{I}\right\|_{1, q}+\left\|\mathbf{I}-\mathbf{A}_{\mathbf{u}}\right\|_{1, q}\right)\left\|\mathbf{F}_{\mathbf{u}}^{-1}\right\|_{1, q} .
\end{aligned}
$$

Since $\left\|\mathbf{F}_{\mathbf{u}}-\mathbf{I}\right\|_{1, q}=\|\nabla \tilde{\mathbf{u}}\|_{1, q} \leq c_{4}\|\mathbf{u}\|_{2, q, \Omega_{0}}$, we just need to show that $\left\|\mathbf{F}_{\mathbf{u}}^{-1}\right\|_{1, q}$ is bounded. From (64), (65), and (66) we obtain, using the Sobolev embedding $W^{1, q}\left(B_{R}\right) \hookrightarrow C_{B}\left(B_{R}\right)$, that

$$
\left\|\mathbf{F}_{\mathbf{u}}^{-1}\right\|_{1, q} \leq c_{5} \mathcal{M}\left(1+\|\nabla \tilde{\mathbf{u}}\|_{1, q}\right) \leq c_{6}\left(\Omega_{0}, \delta, R, K_{0}, q\right)
$$

which together with (69) and (68) implies (62).

The last inequality, (63), can be proved in completely similar fashion.

We can now estimate the perturbation terms in (27). More specifically, we have the following lemma.

Lemma 4.4. Let $q>3$ and $M_{0}$ be as in Lemma 4.3. For $\mathbf{u}_{i} \in S_{q, M}\left(\Omega_{0}\right)$ with $0<M<M_{0}$ and $\left(\mathbf{z}_{i}, \pi_{i}\right) \in \mathcal{X}^{q}(\mathcal{E})(i=1,2)$, the following inequalities hold:

$$
\begin{aligned}
\left\|\mathcal{F}_{\mathbf{u}_{i}}\left(\mathbf{z}_{i}+\mathbf{v}_{\infty}, \pi_{i}\right)\right\|_{\mathcal{Y}^{q}} \leq \\
C_{5} M\left(\left(1+\left|v_{\infty}\right|\right)\left\|\left(\mathbf{z}_{i}, \pi_{i}\right)\right\|_{\mathcal{X}^{q}}+\left\|\left(\mathbf{z}_{i}, \pi_{i}\right)\right\|_{\mathcal{X}^{q}}^{2}\right)
\end{aligned}
$$




$$
\begin{aligned}
& \left\|\mathcal{G}_{\mathbf{u}_{i}}\left(\mathbf{z}_{i}\right)\right\|_{\mathcal{Q}^{q}} \leq C_{5} M\left\|\left(\mathbf{z}_{i}, \pi_{i}\right)\right\|_{\mathcal{X}^{q}}, \\
& \left\|\mathcal{F}_{\mathbf{u}_{1}}\left(\mathbf{z}_{1}+v_{\infty}, \pi_{1}\right)-\mathcal{F}_{\mathbf{u}_{2}}\left(\mathbf{z}_{2}+v_{\infty}, \pi_{2}\right)\right\|_{\mathcal{Y}^{q}} \leq \\
& C_{5}\left(M\left(1+\left|v_{\infty}\right|\right)\left\|\left(\mathbf{z}_{1}-\mathbf{z}_{2}, \pi_{1}-\pi_{2}\right)\right\|_{\mathcal{X}^{q}}+\right. \\
& \left\|\left(\mathbf{z}_{i}, \pi_{i}\right)\right\| \mathcal{X}^{q}\left(1+\left|v_{\infty}\right|\right)\left\|\mathbf{u}_{1}-\mathbf{u}_{2}\right\|_{2, q, \Omega_{0}}+ \\
& \left.M\left\|\mathbf{z}_{i}\right\|_{\mathcal{Z}^{q}}\left\|\mathbf{z}_{1}-\mathbf{z}_{2}\right\|_{\mathcal{Z}^{q}}+\left\|\mathbf{z}_{i}\right\|_{\mathcal{Z}^{q}}^{2}\left\|\mathbf{u}_{1}-\mathbf{u}_{2}\right\|_{2, q, \Omega_{0}}\right), \\
& \left\|\mathcal{G}_{\mathbf{u}_{1}}\left(\mathbf{z}_{1}\right)-\mathcal{G}_{\mathbf{u}_{2}}\left(\mathbf{z}_{2}\right)\right\|_{\mathcal{Q}^{q}} \leq \\
& C_{5}\left(M\left\|\mathbf{z}_{1}-\mathbf{z}_{2}\right\|_{\mathcal{Z}^{q}}+\left\|\mathbf{z}_{i}\right\|_{\mathcal{Z}^{q}}\left\|\mathbf{u}_{1}-\mathbf{u}_{2}\right\|_{2, q, \Omega_{0}}\right),
\end{aligned}
$$

where $C_{5}=C_{5}\left(q, \Omega_{0}, R, \delta, M_{0}\right)$.

Proof. We will only show (72). The other estimates can be shown in a completely similar fashion. First we use the algebraic structure of $W^{1, q}(\mathcal{E})$, Lemma 2.1, and Lemma 4.3 to estimate

$$
\begin{aligned}
\left\|\operatorname{div}\left(\left(\mathbf{I}-\mathbf{A}_{\mathbf{u}_{1}} \mathbf{F}_{\mathbf{u}_{1}}^{-\top}\right) \nabla \mathbf{z}_{1}^{\top}-\left(\mathbf{I}-\mathbf{A}_{\mathbf{u}_{2}} \mathbf{F}_{\mathbf{u}_{2}}^{-\top}\right) \nabla \mathbf{z}_{2}^{\top}\right)\right\|_{q, \mathcal{E}} \\
\leq c_{0}\left(\left\|\mathbf{A}_{\mathbf{u}_{2}} \mathbf{F}_{\mathbf{u}_{2}}^{-\top}-\mathbf{A}_{\mathbf{u}_{1}} \mathbf{F}_{\mathbf{u}_{1}}^{-\top}\right\|_{1, q, \mathcal{E}}\left\|\nabla \mathbf{z}_{1}\right\|_{1, q, \mathcal{E}_{R}}+\right. \\
\left.\quad\left\|\mathbf{I}-\mathbf{A}_{\mathbf{u}_{2}} \mathbf{F}_{\mathbf{u}_{2}}^{-\top}\right\|_{1, q, \mathcal{E}}\left\|\nabla \mathbf{z}_{1}-\nabla \mathbf{z}_{2}\right\|_{1, q, \mathcal{E}_{R}}\right) \\
\leq c_{1}\left(\left\|\mathbf{u}_{1}-\mathbf{u}_{2}\right\|_{2, q, \Omega_{0}}\left\|\mathbf{z}_{1}\right\|_{\mathcal{Z}^{q}}+M\left\|\mathbf{z}_{1}-\mathbf{z}_{2}\right\|_{\mathcal{Z}^{q}}\right) .
\end{aligned}
$$

By similar arguments, and using (59), we furthermore obtain

$$
\begin{aligned}
\left\|\operatorname{div}\left(\left(\pi_{1} \mathbf{I}-\mathbf{A}_{\mathbf{u}_{1}} \pi_{1} \mathbf{I}\right)-\left(\pi_{2} \mathbf{I}-\mathbf{A}_{\mathbf{u}_{2}} \pi_{2} \mathbf{I}\right)\right)\right\|_{q, \mathcal{E}} \leq \\
c_{2}\left(\left\|\mathbf{u}_{1}-\mathbf{u}_{2}\right\|_{2, q, \Omega_{0}}\left\|\pi_{1}\right\|_{\mathcal{P}^{q}}+M\left\|\left(\mathbf{z}_{1}-\mathbf{z}_{2}, \pi_{1}-\pi_{2}\right)\right\|_{\mathcal{X}^{q}}\right) .
\end{aligned}
$$

Next we use the embedding $W^{1, q}\left(B_{R}\right) \hookrightarrow C_{B}\left(B_{R}\right)$, the fact that the support of $\left(\mathbf{I}-\mathbf{A}_{\mathbf{u}_{i}}\right)$ is in $B_{R}$, and Lemma 4.2 to obtain

$$
\begin{gathered}
\left\|\left(\nabla \mathbf{z}_{1}\right)\left(\mathbf{I}-\mathbf{A}_{\mathbf{u}_{1}}\right)\left(\mathbf{z}_{1}+\mathbf{v}_{\infty}\right)-\left(\nabla \mathbf{z}_{2}\right)\left(\mathbf{I}-\mathbf{A}_{\mathbf{u}_{2}}\right)\left(\mathbf{z}_{2}+\mathbf{v}_{\infty}\right)\right\|_{q, \mathcal{E}} \leq \\
c_{3}\left(M\left\|\mathbf{z}_{1}-\mathbf{z}_{2}\right\|_{\mathcal{Z}^{q}}\left(\left\|\mathbf{z}_{1}\right\|_{\mathcal{Z}^{q}}+v_{\infty}\right)+\right. \\
\left\|\mathbf{u}_{1}-\mathbf{u}_{2}\right\|_{2, q, \Omega_{0}}\left\|\mathbf{z}_{1}\right\|_{\mathcal{Z}^{q}}^{2}+ \\
\left\|\mathbf{u}_{1}-\mathbf{u}_{2}\right\|_{2, q, \Omega_{0}}\left\|\mathbf{z}_{1}\right\|_{\mathcal{Z}^{q}} v_{\infty}+ \\
\left.M\left\|\mathbf{z}_{2}\right\|_{\mathcal{Z}^{q}}\left\|\mathbf{z}_{1}-\mathbf{z}_{2}\right\|_{\mathcal{Z}^{q}}\right) .
\end{gathered}
$$

We now need similar estimates as above in the norm $|\cdot|_{-1,12 / 7}$. However, since all the terms on the right-hand side above have bounded support in $B_{R}$, these estimates follow from the ones above since for any $f$ with supp $f \subset B_{R}$ we have $\left|\partial_{i} f\right|_{-1,12 / 7} \leq\|f\|_{12 / 7} \leq C(R)\|f\|_{q}$. Thus, combining all of the above estimates we have shown (72).

With the help of the above lemmas, we are now able to furnish the following existence and uniqueness result for problem (27). 
Theorem 4.5. Let $q>3$ and $M_{0}$ be as in Lemma 4.3. There are constants $J_{i}=J_{i}\left(q, \Omega_{0}, M_{0}, \delta, R, \nu\right)>0(i=1,2)$, such that when $0 \leq M<J_{1}, 0<v_{\infty}<$ $J_{2}$, and $\mathbf{u} \in S_{q, M}\left(\Omega_{0}\right)$, then problem (27) has a unique solution $(\mathbf{z}, \pi) \in \mathcal{X}^{q}(\mathcal{E})$ with

$$
\|(\mathbf{z}, \pi)\|_{\mathcal{X}^{q}} \leq d_{0}\left(v_{\infty}, J_{1}\right),
$$

whereby $d_{0}\left(v_{\infty}, J_{1}\right) \in O\left(v_{\infty}\right)$. Furthermore, for $\mathbf{u}_{1}, \mathbf{u}_{2} \in S_{q, M}\left(\Omega_{0}\right)$ the corresponding solutions $\left(\mathbf{z}_{1}, \pi_{1}\right),\left(\mathbf{z}_{2}, \pi_{2}\right)$ of (27) satisfy

$$
\left\|\left(\mathbf{z}_{1}, \pi_{1}\right)-\left(\mathbf{z}_{2}, \pi_{2}\right)\right\|_{\mathcal{X}^{q}} \leq e\left(v_{\infty}, J_{1}\right)\left\|\mathbf{u}_{1}-\mathbf{u}_{2}\right\|_{2, q, \Omega_{0}},
$$

with $e\left(v_{\infty}, J_{1}\right) \in O\left(v_{\infty}\right)$.

Proof. The proof will be achieved by means of the Caccioppoli-Banach contraction mapping theorem. For $d>0$, set

$$
\mathcal{X}_{d}^{q}(\mathcal{E}):=\left\{(\mathbf{z}, \pi) \in \mathcal{X}^{q}(\mathcal{E}):\|(\mathbf{z}, \pi)\|_{\mathcal{X}^{q}} \leq d\right\},
$$

and consider the map

$$
\mathcal{N}:(\mathbf{Z}, \Pi) \in \mathcal{X}_{d}^{q}(\mathcal{E}) \mapsto(\mathbf{z}, \pi) \in \mathcal{X}_{d}^{q}(\mathcal{E})
$$

where $(\mathbf{z}, \pi)$ is a solution to the problem

$$
\left\{\begin{aligned}
-\nu \Delta \mathbf{z}+v_{\infty} \partial_{1} \mathbf{z}+\nabla \pi & =-(\nabla \mathbf{Z}) \mathbf{Z}+\mathcal{F}_{\mathbf{u}}\left(\mathbf{Z}+\mathbf{v}_{\infty}, \Pi\right) & & \text { in } \mathcal{E} \\
\operatorname{div}(\mathbf{z}) & =\mathcal{G}_{\mathbf{u}}(\mathbf{Z}) & & \text { in } \mathcal{E} \\
\mathbf{z} & =-\mathbf{v}_{\infty} & & \text { on } \Gamma_{0} \\
\lim _{|x| \rightarrow \infty} \mathbf{z} & =0 & &
\end{aligned}\right.
$$

From Lemma 4.1, Lemma 4.2, and Lemma 4.4 we find that problem (76) has a unique solution $(\mathbf{z}, \pi) \in \mathcal{X}^{q}(\mathcal{E})$, whence $\mathcal{N}$ is well-defined. Moreover, by (33), (57), (58), (70), and (71) this solution satisfies the estimate

$$
\begin{aligned}
\|(\mathbf{z}, \pi)\|_{\mathcal{X}^{q}} \leq C_{\Theta}( & \|(\nabla \mathbf{Z}) \mathbf{Z}\|_{\mathcal{Y}^{q}}+\left\|\mathcal{F}_{\mathbf{u}}\left(\mathbf{Z}+v_{\infty}, \Pi\right)\right\|_{\mathcal{Y}^{q}} \\
+ & \left.\left\|\mathcal{G}_{\mathbf{u}}(\mathbf{Z})\right\|_{\mathcal{Q}^{q}}+c_{0} v_{\infty}\right) \\
\leq C_{\Theta}( & \left(C_{1}+C_{2} v_{\infty}^{-1 / 4}\right)\|\mathbf{Z}\|_{\mathcal{Z}^{q}}^{2} \\
& +C_{5} M\left(\left(1+v_{\infty}\right)\|(\mathbf{Z}, \Pi)\|_{\mathcal{X}^{q}}+\|(\mathbf{Z}, \Pi)\|_{\mathcal{X}^{q}}^{2}\right) \\
& \left.+C_{5} M\|(\mathbf{Z}, \Pi)\|_{\mathcal{X}^{q}}+c_{0} v_{\infty}\right) .
\end{aligned}
$$

Choosing $J_{1}, J_{2}<1$ we have both $M<1$ and $v_{\infty}<1$. Thus, putting

$$
\begin{aligned}
& k_{1}\left(v_{\infty}\right):=C_{\Theta}\left(\left(C_{1}+C_{2} v_{\infty}^{-1 / 4}\right)+C_{5}\right) \\
& k_{2}:=3 C_{\Theta} C_{5} \\
& k_{3}:=C_{\Theta} c_{0}
\end{aligned}
$$


we obtain, by (77),

$$
\|(\mathbf{z}, \pi)\|_{\mathcal{X}^{q}} \leq k_{1}\left(v_{\infty}\right) d^{2}+k_{2} J_{1} d+k_{3} v_{\infty} .
$$

It follows that $\mathcal{N}$ maps $\mathcal{X}_{d}^{q}(\mathcal{E})$ into itself if $d$ satisfies

$$
k_{1}\left(v_{\infty}\right) d^{2}+\left(k_{2} J_{1}-1\right) d+k_{3} v_{\infty}<0 .
$$

In order for this inequality to have positive solutions $d$, we must have

$$
k_{2} J_{1}-1<0 \quad \text { and } \quad\left(k_{2} J_{1}-1\right)^{2}>4 k_{1}\left(v_{\infty}\right) k_{3} v_{\infty} .
$$

This is clearly satisfied for $J_{1}$ and $v_{\infty}$ sufficiently small. Now fix such a $J_{1}$. Then for $v_{\infty}$ sufficiently small

$$
d_{0}\left(v_{\infty}, J_{1}\right):=\frac{\left(1-k_{2} J_{1}\right)-\left(1-v_{\infty}\right) \sqrt{\left(k_{2} J_{1}-1\right)^{2}-4 k_{1}\left(v_{\infty}\right) k_{3} v_{\infty}}}{2 k_{1}\left(v_{\infty}\right)}
$$

satisfies (78) and consequently $\mathcal{N}$ maps $\mathcal{X}_{d_{0}}^{q}(\mathcal{E})$ into itself. Clearly $d_{0} \in O\left(v_{\infty}\right)$. We now prove that $\mathcal{N}$ is also a contraction with the above choice of $d_{0}$. For $\left(\mathbf{Z}_{i}, \Pi_{i}\right) \in \mathcal{X}_{d_{0}}^{q}(\mathcal{E}), i=1,2$ we use Lemma 4.1, Lemma 4.2, and Lemma 4.4 to obtain

$$
\begin{aligned}
& \left\|\mathcal{N}\left(\mathbf{Z}_{1}, \Pi_{1}\right)-\mathcal{N}\left(\mathbf{Z}_{2}, \Pi_{2}\right)\right\|_{\mathcal{X}^{q}} \leq \\
& C_{\Theta}\left(\left\|\left(\nabla \mathbf{Z}_{1}\right) \mathbf{Z}_{1}-\left(\nabla \mathbf{Z}_{2}\right) \mathbf{Z}_{2}\right\|_{\mathcal{Y}^{q}}\right. \\
& \quad+\left\|\mathcal{F}_{\mathbf{u}}\left(\mathbf{Z}_{1}+v_{\infty}, \Pi_{1}\right)-\mathcal{F}_{\mathbf{u}}\left(\mathbf{Z}_{2}+v_{\infty}, \Pi_{2}\right)\right\|_{\mathcal{Y}^{q}} \\
& \left.\quad+\left\|\mathcal{G}_{\mathbf{u}}\left(\mathbf{Z}_{1}\right)-\mathcal{G}_{\mathbf{u}}\left(\mathbf{Z}_{2}\right)\right\|_{\mathcal{Q}^{q}}\right) \\
& \quad \quad C_{\Theta}\left(2\left(C_{1}+C_{2} v_{\infty}^{-1 / 4}+C_{5}\right) d_{0}\left(v_{\infty}\right)\left\|\left(\mathbf{Z}_{1}, \Pi_{1}\right)-\left(\mathbf{Z}_{2}, \Pi_{2}\right)\right\|_{\mathcal{X}^{q}}\right. \\
& \left.\quad+3 C_{5} J_{1}\left\|\left(\mathbf{Z}_{1}, \Pi_{1}\right)-\left(\mathbf{Z}_{2}, \Pi_{2}\right)\right\|_{\mathcal{X}^{q}}\right) \\
& \leq\left(2 k_{1}\left(v_{\infty}\right) d_{0}\left(v_{\infty}\right)+k_{2} J_{1}\right)\left\|\left(\mathbf{Z}_{1}, \Pi_{1}\right)-\left(\mathbf{Z}_{2}, \Pi_{2}\right)\right\|_{\mathcal{X}^{q}} .
\end{aligned}
$$

By (79), we have $k_{2} J_{1}<1$. Since furthermore $k_{1}\left(v_{\infty}\right) d_{0}\left(v_{\infty}\right) \rightarrow 0$ as $v_{\infty} \rightarrow 0$, we see that $\mathcal{N}$ becomes a contraction when $v_{\infty}$ is sufficiently small. The existence of a unique fixpoint for $\mathcal{N}$ in $\mathcal{X}_{d_{0}}^{q}(\mathcal{E})$ now follows from the Caccioppoli-Banach Theorem.

We end the proof by showing (75). Consider $\mathbf{u}_{1}, \mathbf{u}_{2} \in S_{q, M}\left(\Omega_{0}\right)$ and corresponding solutions $\left(\mathbf{z}_{1}, p_{1}\right),\left(\mathbf{z}_{2}, p_{2}\right)$ of $(27)$. Estimating as above we obtain

$$
\begin{aligned}
& \left\|\mathcal{N}\left(\mathbf{z}_{1}, \pi_{1}\right)-\mathcal{N}\left(\mathbf{z}_{2}, \pi_{2}\right)\right\|_{\mathcal{X}^{q}} \leq \\
& \leq\left(2 k_{1}\left(v_{\infty}\right) d_{0}\left(v_{\infty}\right)+k_{2} J_{1}\right)\left\|\left(\mathbf{z}_{1}, \pi_{1}\right)-\left(\mathbf{z}_{2}, \pi_{2}\right)\right\|_{\mathcal{X}^{q}+} \\
& \quad C_{\Theta} C_{5}\left(d_{0}\left(v_{\infty}\right)^{2}+2 d_{0}\left(v_{\infty}\right)+d_{0}\left(v_{\infty}\right)\right)\left\|\mathbf{u}_{1}-\mathbf{u}_{2}\right\|_{2, q, \Omega_{0}},
\end{aligned}
$$

which completes the proof, since $\mathcal{N}\left(\mathbf{z}_{1}, \pi_{1}\right)-\mathcal{N}\left(\mathbf{z}_{2}, \pi_{2}\right)=\left(\mathbf{z}_{1}, \pi_{1}\right)-\left(\mathbf{z}_{2}, \pi_{2}\right)$ and $v_{\infty}$ was chosen above such that $2 k_{1}\left(v_{\infty}\right) d\left(v_{\infty}\right)+k_{2} J_{1}<1$. 


\section{The Elasticity Equations}

The objective of this section is to show existence and uniqueness of the elasticity equations. We shall use a fixpoint approach which enables us to easily couple the elasticity equations with the liquid equations.

Consider first the elasticity equations in the case where the elastic body, $\Omega_{0}$, is attached to a nondeformable body $\omega$. In this case the deformation of the elastic body is governed by the traction displacement problem of non-linear elasticity, which we in this section shall treat in the following generality:

$$
\left\{\begin{aligned}
-\operatorname{div}(\boldsymbol{\sigma}(\mathbf{u})) & =\mathbf{F}(\lambda, \mathbf{u}) & & \text { in } \Omega_{0}, \\
\mathbf{n} \boldsymbol{\sigma}(\mathbf{u}) & =\mathbf{G}(\lambda, \mathbf{u}) & & \text { on } \Gamma_{0}, \\
\mathbf{u} & =0 & & \text { on } \Gamma_{1} .
\end{aligned}\right.
$$

We will impose the following conditions on $\mathbf{F}$ and $\mathbf{G}$. We assume that for $\varepsilon, M>$ 0 sufficiently small we can find constants $C_{F}(M), C_{G}(M), D_{F}(M), D_{G}(M)>0$, $\gamma_{F}, \gamma_{G} \in o(M)$, and $\beta_{F}, \beta_{G} \in O(M)$ such that

$$
\begin{aligned}
& \mathbf{F}:(0, \varepsilon) \times S_{q, M}\left(\Omega_{0}\right) \rightarrow L^{q}\left(\Omega_{0}\right), \\
& \|\mathbf{F}(\lambda, \mathbf{u})\|_{q} \leq C_{F}(M) \lambda+\gamma_{F}(M), \\
& \left\|\mathbf{F}\left(\lambda, \mathbf{u}_{1}\right)-\mathbf{F}\left(\lambda, \mathbf{u}_{2}\right)\right\|_{q} \leq\left(D_{F}(M) \lambda+\beta_{F}(M)\right)\left\|\mathbf{u}_{1}-\mathbf{u}_{2}\right\|_{2, q}, \\
& \mathbf{G}:(0, \varepsilon) \times S_{q, M}\left(\Omega_{0}\right) \rightarrow W^{1-1 / q, q}\left(\Gamma_{0}\right), \\
& \|\mathbf{G}(\lambda, \mathbf{u})\|_{1-1 / q, q} \leq C_{G}(M) \lambda+\gamma_{G}(M) \\
& \left\|\mathbf{G}\left(\lambda, \mathbf{u}_{1}\right)-\mathbf{G}\left(\lambda, \mathbf{u}_{2}\right)\right\|_{1-1 / q, q} \leq\left(D_{G}(M) \lambda+\beta_{G}(M)\right)\left\|\mathbf{u}_{1}-\mathbf{u}_{2}\right\|_{2, q} .
\end{aligned}
$$

We have the following theorem of existence and uniqueness of solutions for (81).

Theorem 5.1. Let $q>3$ and assume that $\mathbf{F}$ and $\mathbf{G}$ satisfy (82)-(87). There exists an $M_{1}>0$ such that for all $0<M<M_{1}$ we can find $\lambda_{0}(M)$ such that for all $0<\lambda<\lambda_{0}(M)$ there exists a unique solution $\mathbf{u} \in S_{q, M}\left(\Omega_{0}\right)$ of (81) which furthermore satisfies

$$
\|\mathbf{u}\|_{2, q} \leq C_{6}\left(\|\mathbf{F}(\lambda, \mathbf{u})\|_{q}+\|\mathbf{G}(\lambda, \mathbf{u})\|_{1-1 / q, q}\right),
$$

where $C_{6}=C_{6}\left(M_{1}\right)$.

Proof. Recalling (25), we can write (81) as

$$
\left\{\begin{aligned}
-\operatorname{div}\left(\overline{\boldsymbol{\sigma}}_{\boldsymbol{L}}\right) & =\operatorname{div}\left(\overline{\boldsymbol{\sigma}}_{\boldsymbol{B} \boldsymbol{L}}+\overline{\boldsymbol{\sigma}}_{\boldsymbol{T} \boldsymbol{L}}\right)+\mathbf{F}(\lambda, \mathbf{u}) & & \text { in } \Omega_{0}, \\
\mathbf{n} \overline{\boldsymbol{\sigma}}_{\boldsymbol{L}} & =-\mathbf{n}\left(\overline{\boldsymbol{\sigma}}_{\boldsymbol{B} \boldsymbol{L}}+\overline{\boldsymbol{\sigma}}_{\boldsymbol{T} \boldsymbol{L}}\right)+\mathbf{G}(\lambda, \mathbf{u}) & & \text { on } \Gamma_{0} \\
\mathbf{u} & =0 & & \text { on } \Gamma_{1} .
\end{aligned}\right.
$$

From the theory of linear elasticity it is well known that the operator

$$
\mathcal{L}=\left(-\operatorname{div}\left(\overline{\boldsymbol{\sigma}}_{\boldsymbol{L}}\right), \mathbf{n} \overline{\boldsymbol{\sigma}}_{\boldsymbol{L}}\right)
$$


maps the space

$$
\mathcal{X}^{E}\left(\Omega_{0}\right):=\left\{\mathbf{u} \in W^{2, q}\left(\Omega_{0}\right) \mid \mathbf{u}=0 \text { on } \Gamma_{1}\right\}
$$

homeomorphic onto

$$
\mathcal{Y}^{E}\left(\Omega_{0}\right):=L^{q}\left(\Omega_{0}\right) \times W^{1-1 / q, q}\left(\Gamma_{0}\right)
$$

Now put

$$
\mathcal{T}_{\lambda}(\mathbf{u}):=\mathcal{L}^{-1}\left(\operatorname{div}\left(\overline{\boldsymbol{\sigma}}_{\boldsymbol{B} \boldsymbol{L}}+\overline{\boldsymbol{\sigma}}_{\boldsymbol{T} \boldsymbol{L}}\right)+\mathbf{F}(\lambda, \mathbf{u}),-\mathbf{n}\left(\overline{\boldsymbol{\sigma}}_{\boldsymbol{B} \boldsymbol{L}}+\overline{\boldsymbol{\sigma}}_{\boldsymbol{T} \boldsymbol{L}}\right)+\mathbf{G}(\lambda, \mathbf{u})\right) .
$$

Clearly, a fixpoint of $\mathcal{T}_{\lambda}$ is a solution of (81). We now show that for $M$ sufficiently small we can find $\lambda_{0}(M)$ such that, whenever $0<\lambda<\lambda_{0}(M)$, the mapping $\mathcal{T}_{\lambda}$ maps $\mathcal{X}_{M}^{E}:=\mathcal{X}^{E} \cap \mathrm{B}_{M}(0)$ into itself and is contractive. The existence of a unique fixpoint will then follow from the Caccioppoli-Banach Theorem. To verify that $\mathcal{T}_{\lambda}$ becomes a self-mapping we estimate, using the algebraic structure of $W^{1, q}\left(\Omega_{0}\right)$ and the properties (83) and (86),

$$
\begin{aligned}
& \left\|\mathcal{T}_{\lambda}(\mathbf{u})\right\|_{2, q} \leq \\
& \left\|\mathcal{L}^{-1}\right\|\left(\left\|\operatorname{div}\left(\overline{\boldsymbol{\sigma}}_{\boldsymbol{B} \boldsymbol{L}}(\nabla \mathbf{u}, \nabla \mathbf{u})+\overline{\boldsymbol{\sigma}}_{\boldsymbol{T} \boldsymbol{L}}(\nabla \mathbf{u}, \nabla \mathbf{u}, \nabla \mathbf{u})\right)\right\|_{q}\right. \\
& +\left\|\mathbf{n}\left(\overline{\boldsymbol{\sigma}}_{\boldsymbol{B} \boldsymbol{L}}(\nabla \mathbf{u}, \nabla \mathbf{u})+\overline{\boldsymbol{\sigma}}_{\boldsymbol{T} \boldsymbol{L}}(\nabla \mathbf{u}, \nabla \mathbf{u}, \nabla \mathbf{u})\right)\right\|_{1-1 / q, q} \\
& \left.+\|\mathbf{F}(\lambda, \mathbf{u})\|_{q}+\|\mathbf{G}(\lambda, \mathbf{u})\|_{1-1 / q, q}\right) \\
& \leq\left\|\mathcal{L}^{-1}\right\|\left(c_{1} M^{2}+c_{2} M^{3}+\left(C_{F}+C_{G}\right) \lambda+\gamma_{F}+\gamma_{G}\right) \\
& \leq c_{3}(M) \lambda_{0}(M)+o(M) \text {. }
\end{aligned}
$$

We conclude that $\mathcal{T}_{\lambda}$ becomes a self-mapping on $\mathcal{X}_{M}^{E}$ for $M$ sufficiently small and $\lambda_{0}(M)<\frac{M}{c_{3}(M)}$. Similarly, using now (84) and (87), we estimate

$$
\left\|\mathcal{T}_{\lambda}\left(\mathbf{u}_{1}\right)-\mathcal{T}_{\lambda}\left(\mathbf{u}_{2}\right)\right\|_{2, q} \leq\left(c_{4}(M) \lambda_{0}(M)+O(M)\right)\left\|\mathbf{u}_{1}-\mathbf{u}_{2}\right\|_{2, q}
$$

and conclude that $\mathcal{T}_{\lambda}$ becomes a contraction when $M$ is sufficiently small and $\lambda_{0}(M)<\frac{M}{c_{4}(M)}$. Having established the existence of a unique fixpoint, $\mathbf{u}$, we obtain (88) by an estimate similar to (93).

We now treat the case where the elastic body $\Omega_{0}$ has no non-deformable core, but is held in place by control forces. We then have $\omega=\emptyset$ and $\partial \Omega_{0}=\Gamma_{0}$. In this case we have to solve a free traction problem of the type

$$
\left\{\begin{aligned}
-\operatorname{div}(\boldsymbol{\sigma}(\mathbf{u})) & =\mathbf{F}(\lambda, \mathbf{u}) & & \text { in } \Omega_{0}, \\
\mathbf{n} \boldsymbol{\sigma}(\mathbf{u}) & =\mathbf{G}(\lambda, \mathbf{u}) & & \text { on } \Gamma_{0} .
\end{aligned}\right.
$$

Since we here dealing with a problem with a pure Neumann boundary condition, the problem becomes more delicate than (81) as we have to ensure that the data on the right hand side satisfies compatibility conditions. We thus need to impose additional conditions on $\mathbf{F}$ and $\mathbf{G}$. We again split the first Piola Kirchhoff stress 
tensor, as in (25), into a linear, bi-linear, and tri-linear part, and furthermore put

$$
\mathcal{N}_{E}(\mathbf{u}):=\overline{\boldsymbol{\sigma}}_{B \boldsymbol{L}}(\nabla \mathbf{u}, \nabla \mathbf{u})+\overline{\boldsymbol{\sigma}}_{T \boldsymbol{L}}(\nabla \mathbf{u}, \nabla \mathbf{u}, \nabla \mathbf{u}) .
$$

We need the following additional assumptions on $\mathbf{F}$ and $\mathbf{G}$ :

$$
\begin{aligned}
& \int_{\Omega_{0}} \mathbf{F}(\lambda, \mathbf{u}) \mathrm{d} x+\int_{\Gamma_{0}} \mathbf{G}(\lambda, \mathbf{u}) \mathrm{d} S=0, \\
& \int_{\Omega_{0}} \mathbf{x} \wedge \mathbf{F}(\lambda, \mathbf{u}) \mathrm{d} x+\int_{\Gamma_{0}} \mathbf{x} \wedge \mathbf{G}(\lambda, \mathbf{u}) \mathrm{d} S=\int_{\Omega_{0}}\left(\mathcal{N}_{E}(\mathbf{u})^{\top}-\mathcal{N}_{E}(\mathbf{u})\right)^{\vee} \mathrm{d} x .
\end{aligned}
$$

Here $\mathbf{A}^{\vee} \in \mathbb{R}^{3}$ denotes the adjoint of a skew-symmetric $\mathbf{A} \in \mathbb{R}^{3 \times 3}\left(\mathbf{A}^{\vee}\right.$ is also called the axial vector of $\mathbf{A}$, see [3]). Problem (95) is invariant under infinitesimal ridgid displacements. We shall therefore need the space

$$
\tilde{S}_{q, M}\left(\Omega_{0}\right):=\left\{\mathbf{u} \in S_{q, M}\left(\Omega_{0}\right) \mid \int_{\Omega_{0}} \mathbf{u} \mathrm{d} x=0, \int_{\Omega_{0}} \nabla \mathbf{u} \mathrm{d} x=\int_{\Omega_{0}} \nabla \mathbf{u}^{\top} \mathrm{d} x\right\} .
$$

We can now state the following theorem of existence and uniqueness of solutions for (95).

Theorem 5.2. Let $q>3$ and assume that $\mathbf{F}$ and $\mathbf{G}$ satisfy (82)-(87) and (97)(98). There exists an $M_{2}>0$ such that for all $0<M<M_{2}$ we can find $\lambda_{0}(M)$ such that for all $0<\lambda<\lambda_{0}(M)$ there exists a unique solution $\mathbf{u} \in \tilde{S}_{q, M}\left(\Omega_{0}\right)$ of (95) which furthermore satisfies

$$
\|\mathbf{u}\|_{2, q} \leq C_{7}\left(\|\mathbf{F}(\lambda, \mathbf{u})\|_{q}+\|\mathbf{G}(\lambda, \mathbf{u})\|_{1-1 / q, q}\right),
$$

where $C_{7}=C_{7}\left(M_{2}\right)$.

Proof. Put

$$
\tilde{\mathcal{X}}^{E}\left(\Omega_{0}\right):=\left\{\mathbf{u} \in W^{2, q}\left(\Omega_{0}\right) \mid \int_{\Omega_{0}} \mathbf{u} \mathrm{d} x=0, \int_{\Omega_{0}} \nabla \mathbf{u} \mathrm{d} x=\int_{\Omega_{0}} \nabla \mathbf{u}^{\top} \mathrm{d} x\right\}
$$

and

$$
\begin{aligned}
\tilde{\mathcal{Y}}^{E}\left(\Omega_{0}\right):=\left\{(\mathbf{f}, \mathbf{g}) \in L^{q}\left(\Omega_{0}\right) \times W^{1-1 / q, q}\left(\Gamma_{0}\right) \mid\right. & \\
& \left.\int_{\Omega_{0}} \mathbf{f} \mathrm{d} x+\int_{\Gamma_{0}} \mathbf{g} \mathrm{d} S=0, \int_{\Omega_{0}} \mathbf{x} \wedge \mathbf{f} \mathrm{d} x+\int_{\Gamma_{0}} \mathbf{x} \wedge \mathbf{g} \mathrm{d} S=0\right\} .
\end{aligned}
$$

From the linear theory of elasticity it is well known that $\mathcal{L}$, see $(90)$, maps $\tilde{\mathcal{X}}^{E}\left(\Omega_{0}\right)$ homeomorphic onto $\tilde{\mathcal{Y}}^{E}\left(\Omega_{0}\right)$. We can now replace $\mathcal{X}^{E}$ with $\tilde{\mathcal{X}}^{E}$ and $\mathcal{Y}^{E}$ with $\tilde{\mathcal{Y}}^{E}$ in the proof of Theorem 81 and repeat the proof. Note that assumptions (97)-(98) are necessary in order for $\mathcal{T}_{\lambda}$ to be well defined in the case where $\mathcal{L}$ is considered as an operator from $\tilde{\mathcal{X}}^{E}$ onto $\tilde{\mathcal{Y}}^{E}$. 


\section{Unique Solvability of the Liquid-Structure Problem}

In this section we shall prove the main result of this paper, namely, that the liquid-structure problem, described by the coupled systems (1) and, depending on the model under consideration for the elastic body, (2) or (4), have a locally unique solution if the magnitude of the datum, $v_{\infty}$, is suitably restricted. We shall do so by solving the equivalent systems (27) and (22) or (23), respectively. We shall obtain our result as simple consequence of the theorems in the previous section.

In the case where elastic body is attached to a non-deformable body, we have the following theorem.

Theorem 6.1. Let $q>3$ and $M_{0}$ be the constant from Lemma 4.3. There exists $K_{1}=K_{1}\left(q, \Omega_{0}, \delta, R, M_{0}, \lambda, \mu, \nu\right)>0$ such that, if $0<v_{\infty}<K_{1}$, then the coupled system (27), (22) has a solution $(\mathbf{u},(\mathbf{z}, \pi)) \in W^{2, q}\left(\Omega_{0}\right) \times \mathcal{X}^{q}(\mathcal{E})$ satisfying the inequality

$$
\|\mathbf{u}\|_{2, q, \Omega_{0}}+\|(\mathbf{z}, \pi)\|_{\mathcal{X}^{q}} \leq h,
$$

where $h=h\left(v_{\infty}, q, \Omega_{0}, \delta, R, M_{0}, \lambda, \mu, \nu\right)>0$ and $h \in O\left(v_{\infty}\right)$. Moreover, the solution is unique in $S_{q, M}\left(\Omega_{0}\right) \times \mathcal{X}_{d}^{q}(\mathcal{E}) \subset W^{2, q}\left(\Omega_{0}\right) \times \mathcal{X}^{q}(\mathcal{E})$ for sufficiently small $M>0$ and $d>0$.

Proof. Let $J_{1}$ and $J_{2}$ be as in Theorem 4.5. For $0<M<J_{1}$ we can, by this theorem, associate to any $0<v_{\infty}<J_{2}$ and $\mathbf{u} \in S_{q, M}\left(\Omega_{0}\right)$ a unique solution $(\mathbf{z}, \pi) \in \mathcal{X}^{q}(\mathcal{E})$ of $(27)$ satisfying $(74)$. Thus we can construct a mapping

$$
\begin{aligned}
& \mathbf{G}:\left(0, J_{2}\right) \times S_{q, M}\left(\Omega_{0}\right) \rightarrow W^{1-1 / q, q}\left(\Gamma_{0}\right), \\
& \mathbf{G}\left(v_{\infty}, \mathbf{u}\right):=\mathbf{n} \mathbf{T}_{F}^{\mathbf{u}}(\mathbf{z}, \pi) .
\end{aligned}
$$

We now verify that $\mathbf{G}$ satisfies (86)-(87). Using the boundedness of the trace operator

$$
\operatorname{Tr}_{R}: W^{1, q}\left(\mathcal{E}_{R}\right) \rightarrow W^{1-1 / q, q}\left(\Gamma_{0}\right),
$$

the algebraic structure of $W^{1, q}(\mathcal{E})$, Lemma 4.3 , and property (59), we obtain

$$
\begin{aligned}
& \left\|\mathbf{n} \mathbf{T}_{F}^{\mathbf{u}}(\mathbf{z}, \pi)\right\|_{1-1 / q, q, \Gamma_{0}}=\left\|\mathbf{n} \mathbf{A}_{\mathbf{u}}\left(2 \nu \mathbf{D}_{\mathbf{u}}(\mathbf{z})-\pi \mathbf{I}\right)\right\|_{1-1 / q, q, \Gamma_{0}} \\
& \quad \leq c_{0}\left\|\mathbf{A}_{\mathbf{u}}\left(2 \nu \mathbf{D}_{\mathbf{u}}(\mathbf{z})-\pi \mathbf{I}\right)\right\|_{1, q, \mathcal{E}_{R}} \\
& \quad \leq c_{1}\left(1+C_{3} M\right)\left(\|\pi\|_{1, q, \mathcal{E}_{R}}+\left(1+C_{3} M\right)\|\mathbf{z}\|_{2, q, \mathcal{E}_{R}}\right) \\
& \quad \leq c_{2}(M)\|(\mathbf{z}, \pi)\|_{\mathcal{X}^{q}} .
\end{aligned}
$$

Since $(\mathbf{z}, \pi)$ satisfies (74), we conclude that $\mathbf{G}$ satisfies (86). Similarly, we estimate

$$
\begin{aligned}
& \left\|\mathbf{n} \mathbf{T}_{F}^{\mathbf{u}_{1}}\left(\mathbf{z}_{1}, \pi_{1}\right)-\mathbf{n} \mathbf{T}_{F}^{\mathbf{u}_{2}}\left(\mathbf{z}_{2}, \pi_{2}\right)\right\|_{1-1 / q, q, \Gamma_{0}} \\
& \quad \leq c_{3}(M)\left(\left\|\left(\mathbf{z}_{1}, \pi_{1}\right)-\left(\mathbf{z}_{2}, \pi_{2}\right)\right\|_{\mathcal{X}^{q}}+\left\|\mathbf{u}_{1}-\mathbf{u}_{2}\right\|_{2, q, \Omega_{0}}\left\|\left(\mathbf{z}_{i}, \pi_{i}\right)\right\|_{\mathcal{X}^{q}}\right)
\end{aligned}
$$


and conclude by (74) and (75) that $\mathbf{G}$ satisfies (87). We now apply Theorem 5.1 with $\mathbf{G}$ as above and $\mathbf{F}:=0$ and obtain a unique solution $\mathbf{u} \in S_{q, M}\left(\Omega_{0}\right)$ of (81) for sufficiently small $M$ and $v_{\infty}$. Clearly this solution together with the corresponding solution of (27) is a solution of the coupled systems (27), (22). Moreover, by (88), (101), and (74) it satisfies (100). Finally, we note that any other solution of (27), (22) also solves (81) with the same choice of $\mathbf{F}$ and $\mathbf{G}$ as above. Hence local uniqueness follows from Theorem 5.1 and Theorem 4.5.

We now move on to the case where the elastic body is held in place by a control forces. We have following theorem.

Theorem 6.2. Let $q>3$ and $M_{0}$ be the constant from Lemma 4.3. There exists $K_{2}=K_{2}\left(q, \Omega_{0}, \delta, R, M_{0}, \lambda, \mu, \nu\right)>0$ such that, if $0<v_{\infty}<K_{2}$, then the coupled system (27), (23) has a solution $(\mathbf{c}, \mathbf{k}, \mathbf{u},(\mathbf{z}, \pi)) \in \mathbb{R}^{3} \times \mathbb{R}^{3} \times W^{2, q}\left(\Omega_{0}\right) \times \mathcal{X}^{q}(\mathcal{E})$ satisfying the inequality

$$
\|\mathbf{u}\|_{2, q, \Omega_{0}}+\|(\mathbf{z}, \pi)\| \mathcal{X}^{q}+|\mathbf{c}|+|\mathbf{k}| \leq l,
$$

where $l=l\left(v_{\infty}, q, \Omega_{0}, \delta, R, M_{0}, \lambda, \mu, \nu\right)>0$ and $l \in O\left(v_{\infty}\right)$. Moreover, the solution is unique in $\mathbb{R}^{3} \times \mathbb{R}^{3} \times S_{q, M}\left(\Omega_{0}\right) \times \mathcal{X}_{d}^{q}(\mathcal{E})$ for sufficiently small $M>0$ and $d>0$.

Proof. Without loss of generality, we assume $\int_{\Omega_{0}} \mathbf{x} \mathrm{d} x=0$. Let $J_{1}$ and $J_{2}$ be as in Theorem 4.5. For $0<M<J_{1}$ we can, by this theorem, associate to any $0<v_{\infty}<J_{2}$ and $\mathbf{u} \in S_{q, M}\left(\Omega_{0}\right)$ a unique solution $(\mathbf{z}, \pi) \in \mathcal{X}^{q}(\mathcal{E})$ of $(27)$ satisfying (74). Now define

$$
\begin{aligned}
& \mathbf{F}:\left(0, J_{2}\right) \times S_{q, M}\left(\Omega_{0}\right) \rightarrow W^{2, q}\left(\Omega_{0}\right), \\
& \mathbf{F}\left(v_{\infty}, \mathbf{u}\right):=\frac{-1}{\left|\Omega_{0}\right|} \int_{\Gamma_{0}} \mathbf{n} \mathbf{T}_{F}^{\mathbf{u}}(\mathbf{z}, \pi) \mathrm{d} S
\end{aligned}
$$

and

$$
\begin{aligned}
& \mathbf{G}:\left(0, J_{2}\right) \times S_{q, M}\left(\Omega_{0}\right) \rightarrow W^{1-1 / q, q}\left(\Gamma_{0}\right) \\
& \mathbf{G}\left(v_{\infty}, \mathbf{u}\right):=\frac{1}{2\left|\Omega_{0}\right|} \mathbf{k}\left(\mathbf{u}, v_{\infty}\right) \wedge \mathbf{n}+\mathbf{n} \mathbf{T}_{F}^{\mathbf{u}}(\mathbf{z}, \pi)
\end{aligned}
$$

with

$$
\mathbf{k}\left(\mathbf{u}, v_{\infty}\right):=\int_{\Omega_{0}}\left(\mathcal{N}_{E}(\mathbf{u})^{\top}-\mathcal{N}_{E}(\mathbf{u})\right)^{\vee} \mathrm{d} x-\int_{\Gamma_{0}} \mathbf{x} \wedge\left(\mathbf{n} \mathbf{T}_{F}^{\mathbf{u}}(\mathbf{z}, \pi)\right) \mathrm{d} S
$$

We now verify that $\mathbf{F}$ and $\mathbf{G}$ satisfy the conditions (82)-(87) and (97)-(98). By estimates similar to (101) and (102), we easily verify that $\mathbf{F}$ meets conditions (83)-(84). In a similar manner we can also deduce

$$
\begin{aligned}
\left\|\mathbf{G}\left(\mathbf{u}, v_{\infty}\right)\right\|_{1-1 / q, q} & \leq c_{0}\left|\mathbf{k}\left(\mathbf{u}, v_{\infty}\right)\right|+c_{1}(M) v_{\infty} \\
& \leq c_{2}\left(M^{3}+M^{2}\right)+c_{3}(M) v_{\infty}
\end{aligned}
$$


and

$$
\begin{aligned}
& \left\|\mathbf{G}\left(\mathbf{u}_{1}, v_{\infty}\right)-\mathbf{G}\left(\mathbf{u}_{2}, v_{\infty}\right)\right\|_{1-1 / q, q} \\
& \quad \leq c_{4}\left|\mathbf{k}\left(\mathbf{u}_{1}, v_{\infty}\right)-\mathbf{k}\left(\mathbf{u}_{2}, v_{\infty}\right)\right|+c_{5}(M) v_{\infty}\left\|\mathbf{u}_{1}-\mathbf{u}_{2}\right\|_{2, q} \\
& \quad \leq c_{6}\left(M^{2}+M\right)\left\|\mathbf{u}_{1}-\mathbf{u}_{2}\right\|_{2, q}+c_{5}(M) v_{\infty}\left\|\mathbf{u}_{1}-\mathbf{u}_{2}\right\|_{2, q}
\end{aligned}
$$

and thus verify that $\mathbf{G}$ satisfies (86)-(87). Finally, we note that, by construction, F and $\mathbf{G}$ satisfy (97)-(98). This follows from the identity

$$
\mathbf{a}=\frac{1}{2|\Omega|} \int_{\Gamma_{0}} \mathbf{x} \wedge(\mathbf{a} \wedge \mathbf{n}) \mathrm{d} S, \quad \forall \mathbf{a} \in \mathbb{R}^{3}
$$

and the assumption that $\int_{\Omega_{0}} \mathbf{x} \mathrm{d} x=0$. We can now apply Theorem 5.2, with $\mathbf{F}$ and $\mathbf{G}$ as above, and obtain a solution, $\mathbf{u}$, of $(95)$. Let $(\mathbf{z}, \pi)$ denote the corresponding solution of (27) and put

$$
\mathbf{c}:=\frac{-1}{\left|\Omega_{0}\right|} \int_{\Gamma_{0}} \mathbf{n} \mathbf{T}_{F}^{\mathbf{u}}(\mathbf{z}, \pi) \mathrm{d} S
$$

and

$$
\mathbf{k}:=\mathbf{k}\left(\mathbf{u}, v_{\infty}\right)=\int_{\Omega_{0}}\left(\mathcal{N}_{E}(\mathbf{u})^{\top}-\mathcal{N}_{E}(\mathbf{u})\right)^{\vee} \mathrm{d} x-\int_{\Gamma_{0}} \mathbf{x} \wedge\left(\mathbf{n} \mathbf{T}_{F}^{\mathbf{u}}(\mathbf{z}, \pi)\right) \mathrm{d} S
$$

Clearly $(\mathbf{c}, \mathbf{k}, \mathbf{u},(\mathbf{z}, \pi))$ is a solution of the coupled system (27), (23). The bound (103) follows as in the proof of Theorem 6.1. Finally, we note that any other solution of (27), (23) satisfies (95) with the above choice of $\mathbf{F}$ and $\mathbf{G}$. This can easily be seen by computing the compatibility conditions of (23). Local uniqueness is therefore a consequence of Theorem 5.2 and Theorem 4.5.

Remark 6.3. The solutions of the Navier-Stokes equations found in Theorem 6.1 and Theorem 6.2 are physically reasonable in the sense of Finn. This follows from the fact that the solutions, by construction, belong to $D^{1,2}$ and hence are so-called D-solutions.

Remark 6.4. We could also treat other types of control forces in Theorem 6.2 than the ones chosen in (23). For example, instead of looking for a control surface force acting tangential to the normal of the reference domain surface, we could set out to find a control surface force acting tangential to the normal in the deformed domain. Such a force would be of type

$$
\mathbf{g}=\mathbf{k} \wedge\left(\mathbf{A}_{\mathbf{u}}^{\top} \mathbf{n}\right) \text { on } \partial \Omega_{0} .
$$

Since $\mathbf{A}_{\mathbf{u}}$ is close to the identity for small $\mathbf{u}$, our method of proof for Theorem 6.2 would yield the same result of existence and local uniqueness in this case.

Acknowledgment. The work of G.P. GALDI was partially supported by NSF Grant DMS-0707281. 


\section{References}

[1] Stuart S. Antman and Massimo Lanza de Cristoforis. Nonlinear, nonlocal problems of fluid-solid interactions. Degenerate diffusions (Minneapolis, MN, 1991), 1-18, IMA Vol. Math. Appl., 47, Springer, New York. 1993

[2] Carlos Brebbia and Subrata K. Chakrabarti (Eds.) Fluid structure interaction. WITpress, Vol. 56. 2001

[3] P. Chadwick. Continuum Mechanics, Concise Theory and Problems. Dover Publ. Inc., 1999.

[4] Antonin Chambolle, Benoît Desjardins, Maria J. Esteban and Céline Grandmont. Existence of weak solutions for the unsteady interaction of a viscous fluid with an elastic plate. J. Math. Fluid Mech. 7(3): 368-404, 2005

[5] Philippe G. Ciarlet. Mathematical elasticity. Volume I: Three-dimensional elasticity. North Holland, 1988.

[6] Daniel Coutand and Steve Shkoller. Motion of an elastic solid inside an incompressible viscous fluid. Arch. Ration. Mech. Anal., 176(1):25-102, 2005.

[7] Daniel Coutand and Steve Shkoller. The interaction between quasilinear elastodynamics and the Navier-Stokes equations. Arch. Ration. Mech. Anal., 179(3):303-352, 2006.

[8] Giovanni P. Galdi. An introduction to the mathematical theory of the Navier-Stokes equations. Vol. 1: Linearized steady problems. Springer Tracts in Natural Philosophy., 1994.

[9] Giovanni P. Galdi. An introduction to the mathematical theory of the Navier-Stokes equations. Vol. II: Nonlinear steady problems. Springer Tracts in Natural Philosophy., 1994.

[10] Giovanni P. Galdi. Further properties of steady-state solutions to the Navier-Stokes problem past a three-dimensional obstacle. J. Math. Phys. 48(6): 065207, 43 pp, 2007

[11] Giovanni P. Galdi, Anne M. Robertson, Rolf Rannacher and Stefan Turek. Hemodynamical Flows: Modeling, Analysis and Simulation. Oberwolfach Seminar Series Vol. 35, Birkhuser-Verlag. 2008

[12] Céline Grandmont. Existence for a three-dimensional steady-state fluidstructure interaction problem. J. Math. Fluid Mech., 4(1):76-94, 2002.

[13] Céline Grandmont and Yvon Maday. Fluid-structure interaction: a theoretical point of view. Fluid-structure interaction. 1-22, Innov. Tech. Ser., Kogan Page Sci., London. 2003. 
[14] Leonid V. Kantorovich and Gleb P. Akilov. Functional analysis in normed spaces. Pergamon Press., 1964.

[15] Massimo Lanza de Cristoforis and Stuart S. Antman. The large deformation of nonlinearly elastic tubes in two-dimensional flows. SIAM J. Math. Anal. $22(5)$ 1193-1221, 1991

[16] Massimo Lanza de Cristoforis and Stuart S. Antman. The large deformation of non-linearly elastic shells in axisymmetric flows. Ann. Inst. H. Poincar Anal. Non Linaire. 9(4):433-464, 1992

[17] Martin Rumpf. On equilibria in the interaction of fluids and elastic solids. Heywood, J. G. (ed.) et al., Theory of the Navier-Stokes equations. Proceedings of the third international conference on the Navier-Stokes equations: theory and numerical methods, Oberwolfach, Germany, June 5-11, 1994. Singapore: World Scientific. Ser. Adv. Math. Appl. Sci. 47, 136-158 (1998).

[18] Christina Surulescu. On the stationary interaction of a Navier-Stokes fluid with an elastic tube wall. Appl. Anal. 86(2):149-165, 2007 